\title{
More Efficient Universal Circuit Constructions
}

\author{
Daniel Günther, Ágnes Kiss ${ }^{(凶)}$, and Thomas Schneider \\ TU Darmstadt, Darmstadt, Germany \\ guenther@rangar.de, \{agnes.kiss, thomas.schneider\}@crisp-da.de
}

\begin{abstract}
A universal circuit (UC) can be programmed to simulate any circuit up to a given size $n$ by specifying its program bits. UCs have several applications, including private function evaluation (PFE). The asymptotical lower bound for the size of a UC is proven to be $\Omega(n \log n)$. In fact, Valiant (STOC'76) provided two theoretical UC constructions using so-called 2-way and 4-way constructions, with sizes $5 n \log _{2} n$ and $4.75 n \log _{2} n$, respectively. The 2 -way UC has recently been brought into practice in concurrent and independent results by Kiss and Schneider (EUROCRYPT'16) and Lipmaa et al. (Eprint 2016/017). Moreover, the latter work generalized Valiant's construction to any $k$-way UC.

In this paper, we revisit Valiant's UC constructions and the recent results, and provide a modular and generic embedding algorithm for any $k$-way UC. Furthermore, we discuss the possibility for a more efficient $\mathrm{UC}$ based on a 3 -way recursive strategy. We show with a counterexample that even though it is a promising approach, the 3 -way UC does not yield an asymptotically better result than the 4 -way UC. We propose a hybrid approach that combines the 2 -way with the 4 -way UC in order to minimize the size of the resulting UC. We elaborate on the concrete size of all discussed UC constructions and show that our hybrid UC yields on average $3.65 \%$ improvement in size over the 2 -way UC. We implement the 4-way UC in a modular manner based on our proposed embedding algorithm, and show that our methods for programming the UC can be generalized for any $k$-way construction.
\end{abstract}

Keywords: Universal circuit $\cdot$ Private function evaluation $\cdot$ Function hiding

\section{Introduction}

Universal circuits (UCs) are Boolean circuits that can be programmed to simulate any Boolean function $f(x)$ up to a given size by specifying a set of program bits $p_{f}$. The UC then receives these program bits as input besides the input $x$ to the functionality, and computes the result as $U C\left(x, p_{f}\right)=f(x)$. This means that the same UC can evaluate multiple Boolean circuits, only the different program bits are to be specified.

Valiant proposed an asymptotically size-optimal construction in [Val76] with size $\Theta(n \log n)$ and depth $\mathcal{O}(n)$, where $n$ is the size of the simulated Boolean circuit description of $f(x)$. He provides two constructions, based on 2-way and

(C) International Association for Cryptologic Research 2017

T. Takagi and T. Peyrin (Eds.): ASIACRYPT 2017, Part II, LNCS 10625, pp. 443-470, 2017.

https://doi.org/10.1007/978-3-319-70697-9_16 
4-way recursive structures. Recently, optimizations of Valiant's size-optimized construction appeared in concurrent and independent works of [KS16] and [LMS16]. Both works implement Valiant's 2-way recursive construction.

\subsection{Applications of Universal Circuits}

Size-optimized universal circuits have many applications. We review some of them here and refer to [KS16,LMS16] for further details.

Private Function Evaluation (PFE). Secure two-party computation or secure function evaluation (SFE) provides interactive protocols for evaluating a public function $f(x, y)$ on two parties' private inputs $x$ and $y$. However, in some scenarios, the function $f$ is a secret input of one of the parties. This setting is called private function evaluation (PFE). PFE of $f(x)$ can be achieved by running SFE of $U C\left(x, p_{f}\right)$, where the UC is a public function and the program bits $p_{f}$ - and therefore $f$ - are kept private due to the properties of SFE. Protocols designed especially for PFE such as [MS13,BBKL17] achieve the same asymptotic complexity $\mathcal{O}(n \log n)$ as PFE using UCs, where $n$ is the size of the function $f .{ }^{1}$ However, to the best of our knowledge, they have not yet been implemented, and they are not as generally applicable as PFE with UCs.

UC-based PFE can be easily integrated into any SFE framework and can directly benefit from recent optimizations. For instance, outsourcing UC-based PFE is directly possible with outsourced SFE [KR11]. The non-interactive secure computation protocol of [AMPR14] can also be generalized to obtain a noninteractive PFE protocol [LMS16].

One of the first applications for PFE was privacy-preserving checking for credit worthiness [FAZ05], where not only the loanee's data, but also the loaner's function needs to be kept private. PFE allows for running proprietary software on private data, such as privacy-preserving software diagnosis [BPSW07], medical programs $[\mathrm{BFK}+09]$, or privacy-preserving intrusion detection [NSMS14]. UCs can be applied to obliviously filter remote streaming data [OI05] and for hiding queries in private database management systems such as Blind Seer $[\mathrm{PKV}+14$, FVK+15].

Applications Beyond PFE. Universal circuits can be applied for program obfuscation. Candidates for indistinguishability obfuscation are constructed using a UC as a building block in [GGH+13a,BOKP15], which can be improved using Valiant's UC implementation [KS16]. Direct program obfuscation was proposed in [Zim15], where the circuit is a secret key to a UC. [LMS16] mentions that UCs can be applied for secure two-party computation in the batch execution setting $[\mathrm{HKK}+14, \mathrm{LR} 15]$. It can be applied for verifiable computation [FGP14], and

\footnotetext{
${ }^{1}$ There also exist PFE protocols with linear complexity $\mathcal{O}(n)$ which are based on public-key primitives [KM11, MS13, MSS14]. However, the concrete complexity of these protocols is worse than that of the protocols based on (mostly) symmetric-key primitives, i.e., the OT-based PFE protocols of [MS13, BBKL17] or PFE using UCs.
} 
for multi-hop homomorphic encryption [GHV10]. Ciphertext-policy AttributeBased Encryption was proposed in [Att14], where the policy circuit is hidden $[\mathrm{GGH}+13 \mathrm{~b}]$.

\subsection{Related Work on Universal Circuits}

Valiant defined universal circuits in [Val76] and gave two size-optimized constructions. The constructions are based on so-called edge-universal graphs (EUGs) and utilize either a 2-way or a 4-way recursive structure, also called 2-way or 4-way UCs. Both achieve the asymptotically optimal size $\Theta(n \log n)$ [Val76, Weg87], where $n$ is the size of the simulated circuit. The concrete complexity of the 4 -way UC is $\sim 4.75 n \log _{2} n$ which is smaller than that of the 2-way UC of $\sim 5 n \log _{2} n$ [Val76].

The first modular UC construction was proposed by Kolesnikov and Schneider in [KS08b]. This construction achieves a non-optimal asymptotic complexity of $\mathcal{O}\left(n \log ^{2} n\right)$, and was the first implementation of UCs. A generalization of UCs for $n$-input gates was given in [SS08].

Recently, two independent works have optimized and implemented Valiant's 2-way UC [KS16,LMS16]. Kiss and Schneider in [KS16] mainly focus on the most prominent application of UCs, i.e., private function evaluation (PFE). Due to the free-XOR optimization of [KS08a] in the SFE setting, they optimize the size of the UC for the number of AND gates in the resulting UC implementation and provide a framework for PFE using UCs as public function. They also propose hybrid constructions for circuits with a large number of inputs and outputs, utilizing efficient building blocks from [KS08b]. Lipmaa et al. in [LMS16] also provide an (unpublished) implementation of the 2-way UC. While keeping the number of AND gates minimal, they additionally optimize for the total number of gates, i.e., include optimizations to also reduce the number of XOR gates. They adapt the construction to arithmetic circuits and generalize the design to a $k$-way construction in a modular manner, for $k \geq 2$.

Both papers utilize 2-coloring of the underlying graphs for defining the program bits $p_{f}$ for any given functionality $f$. Generally, 2-coloring can be utilized for any $2^{i}$-way construction. [LMS16] calculate the optimal value for $k$ to be 3.147 , and conclude that the two candidates for the most efficient $2^{i}$-way constructions are the 2-way and 4-way UCs, of which the 4-way construction results in an asymptotically smaller size.

So far only Valiant's 2-way UC has been implemented and the not yet implemented 4-way UC was postulated to be the most efficient one.

\subsection{Outline and Our Contributions}

In summary, we provide the first implementation and detailed evaluation of Valiant's 4-way UC and propose an even more efficient hybrid UC. We elaborate on the size of the generalized $k$-way UCs for $k \neq 2$ and $k \neq 4$.

After revisiting Valiant's UC construction [Val76,KS16] and its $k$-way generalization [LMS16] in Sect.2, we provide the following contributions: 
Our modular programming algorithm (Sect.3): We detail a modular algorithm that provides the description of the input function $f$ as program bits $p_{f}$ to the UC, both for Valiant's 4-way UC as well as for the $k$-way $\mathrm{UC}$ of Lipmaa et al. [LMS16].

New universal circuit constructions (Sect.4): We start with a new 3-way UC. After providing modular building blocks for this UC, we show that it is asymptotically larger than Valiant's UCs. Then, we propose a hybrid UC construction that can efficiently combine $k$-way constructions for multiple values of $k .^{2}$ With this, we combine Valiant's 2-way and 4-way UCs to achieve the smallest UC known so far.

Size of UCs (Sect. 5): We compare the asymptotic and concrete sizes of Valiant's (2-way and 4 -way) UCs and that of different $k$-way UCs. We show that of all $k$-way UCs, Valiant's 4-way UC provides the best results for large circuits. Moreover, our hybrid UC in most cases improves over the 2 -way UC by up to around $4.5 \%$ in its size, and over the 4 -way UC by up to $2 \%$ (for large input circuits). In Table 1 we compare the concrete communication of PFE using SFE and our new UC implementation to the previous works on special-purpose OT-based PFE protocols.

Implementation of Valiant's 4-way UC and experiments (Sect.6): We implement Valiant's 4-way UC and describe how our implementation can directly be used in the PFE framework of [KS16]. We experimentally evaluate the performance of our UC generation and programming algorithm with a set of example circuits and compare it on the same platform with the 2-way UC compiler of [KS16].

Table 1. Comparison of overall communication between special-purpose PFE protocols and UC-based ones for simulated circuits of size $n$. The numbers are for 128 bit symmetric security. The underlying SFE protocol for UC-based PFE is Yao's protocol [Yao86] with the garbled row reduction optimization [NPS99] and X- and Y-switching blocks are instantiated using free XORs as described in [KS08a]. This yields one ciphertext per $\mathrm{X}$ - and Y-switching block, and three ciphertexts per universal gate.

\begin{tabular}{l||r|r||r|r|r}
\multicolumn{1}{c||}{$n$} & \multicolumn{2}{c||}{ Special-purpose PFE } & \multicolumn{3}{c}{ UC-based PFE using Yao } \\
\cline { 2 - 7 } & {$[\mathrm{MS} 13]$} & [BBKL17] & 2-way UC [KS16] & Our 4-way UC & Our hybrid UC \\
\hline \hline $10^{3}$ & $3.5 \mathrm{MB}$ & $2.0 \mathrm{MB}$ & $0.6 \mathrm{MB}$ & $0.6 \mathrm{MB}$ & $0.6 \mathrm{MB}$ \\
$10^{4}$ & $44.8 \mathrm{MB}$ & $26.3 \mathrm{MB}$ & $8.4 \mathrm{MB}$ & $8.4 \mathrm{MB}$ & $8.2 \mathrm{MB}$ \\
$10^{5}$ & $549.6 \mathrm{MB}$ & $324.0 \mathrm{MB}$ & $109.6 \mathrm{MB}$ & $107.8 \mathrm{MB}$ & $106.2 \mathrm{MB}$ \\
$10^{6}$ & $6509.9 \mathrm{MB}$ & $3847.9 \mathrm{MB}$ & $1360.3 \mathrm{MB}$ & $1308.4 \mathrm{MB}$ & $1308.4 \mathrm{MB}$ \\
$10^{7}$ & $75236.5 \mathrm{MB}$ & $44562.1 \mathrm{MB}$ & $16038.8 \mathrm{MB}$ & $15677.7 \mathrm{MB}$ & $15413.7 \mathrm{MB}$
\end{tabular}

\footnotetext{
${ }^{2}$ Our hybrid UC is orthogonal to that of [KS16], who combine Valiant's UC with building blocks from [KS08b] for the inputs and outputs.
} 


\section{Preliminaries}

In this section, we summarize the existing UC constructions. We provide necessary background information in Sect. 2.1, explain Valiant's construction [Val76] in Sect. 2.2 and the improvements of [KS16,LMS16] on the 2-way, 4 -way and $k$-way UCs in Sects. 2.3, 2.4 and 2.5, respectively.

\subsection{Preliminaries to Valiant's UC Constructions}

Let $G=(V, E)$ be a directed graph with set of nodes $V$ and edges $E \subseteq V \times V$. The number of incoming [outgoing] edges of a node is called its indegree [outdegree]. A graph has fanin [fanout] $d$ if the indegree [outdegree] of all its nodes is at most $d$. In the following, we denote by $\Gamma_{d}(n)$ the set of all acyclic graphs with fanin and fanout $d$ having $n$ nodes. Similarly, the fanin [fanout] of a circuit can be defined based on the maximal number of incoming [outgoing] wires of all its gates, inputs and outputs.

Let $G=(V, E) \in \Gamma_{d}(n)$. A mapping $\eta^{G}: V \rightarrow\{1, \ldots, n\}$ is called topological order if $\left(a_{i}, a_{j}\right) \in E \Rightarrow \eta^{G}\left(a_{i}\right)<\eta^{G}\left(a_{j}\right)$ and $\forall a_{1}, a_{2} \in V: \eta^{G}\left(a_{1}\right)=\eta^{G}\left(a_{2}\right) \Rightarrow$ $a_{1}=a_{2}$. A topological order in $G \in \Gamma_{d}(n)$ can be found with computational complexity $\mathcal{O}(d n)$.

A circuit $C_{u, v}^{k^{*}}$ with $u$ inputs, $k^{*}$ gates and $v$ outputs and fanin or fanout $d>2$ can be reduced to a circuit with fanin and fanout 2. Shannon's expansion theorem [Sha49,Sch08] describes how gates with larger fanin can be reduced to gates with two inputs by adding additional gates. [Val76, KS16] describe adding copy gates in order to eliminate larger fanout and elaborate on the implied overhead $\left(k \leq 2 k^{*}+v\right)$. [KS08b, KS16] implement these methods and we thus assume that our input Boolean circuit $C_{u, v}^{k}$ has fanin and fanout 2 for all its $u$ inputs, $k$ gates and $v$ outputs. We transform $C_{u, v}^{k}$ into a $\Gamma_{2}(n)$ graph $G$ with $n=u+v+k$ by creating a node for each input, gate and output, and an edge for each wire in $C_{u, v}^{k}$.

Edge-embedding is a mapping from graph $G=(V, E)$ into $G^{\prime}=\left(V^{\prime}, E^{\prime}\right)$ with $V \subseteq V^{\prime}$ and $E^{\prime}$ containing a path for each $e \in E$, such that the paths are pairwise edge-disjoint. A graph $U_{n}\left(\Gamma_{d}\right)=\left(V_{U}, E_{U}\right)$ is an Edge-Universal Graph (EUG) for $\Gamma_{d}(n)$ if every graph $G \in \Gamma_{d}(n)$ can be edge-embedded into $U_{n}\left(\Gamma_{d}\right) .^{3}$ $U_{n}\left(\Gamma_{d}\right)$ has distinguished nodes called poles $\left\{p_{1}, \ldots, p_{n}\right\} \subseteq V_{U}$ where each node $a \in V$ is mapped to exactly one pole with a mapping $\varphi$, such that every node in $G$ has a corresponding pole in $U_{n}\left(\Gamma_{d}\right)$. This mapping is defined by a concrete topological order $\eta^{G}$ of the original graph $G$, i.e., $\varphi: V \rightarrow V_{U}$ with $\varphi(a)=p_{\eta^{G}(a)}$. Besides the poles, $U_{n}\left(\Gamma_{d}\right)$ might have additional nodes that enable the edgeembedding. For each edge $\left(a_{i}, a_{j}\right) \in E$ we then define a disjoint path between the corresponding poles $\left(\varphi\left(a_{i}\right), \ldots, \varphi\left(a_{j}\right)\right)=\left(p_{\eta^{G}\left(a_{i}\right)}, \ldots, p_{\eta^{G}\left(a_{j}\right)}\right)$ in $U_{n}\left(\Gamma_{d}\right)$, i.e., without using any edge in $U_{n}\left(\Gamma_{d}\right)$ in more than one path.

Let $U_{n}\left(\Gamma_{1}\right)$ be an EUG for graphs in $\Gamma_{1}(n)$ with poles $P=\left\{p_{1}, \ldots, p_{n}\right\}$. The poles have fanin and fanout 1 , while all other nodes have fanin and fanout 2 . An

\footnotetext{
${ }^{3}$ For the sake of simplicity, we denote this graph with $U_{n}\left(\Gamma_{d}\right)$ instead of $U\left(\Gamma_{d}(n)\right)$.
} 


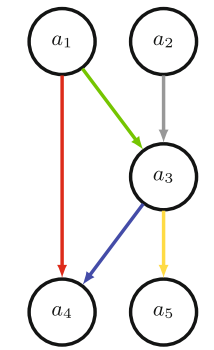

(a) $\Gamma_{2}(5)$ graph $G$

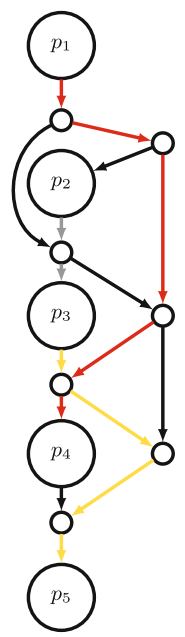

(b) $U_{5}\left(\Gamma_{1}\right)_{1}$

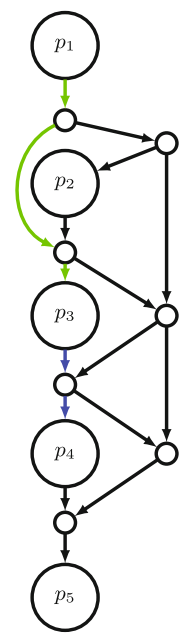

(c) $U_{5}\left(\Gamma_{1}\right)_{2}$

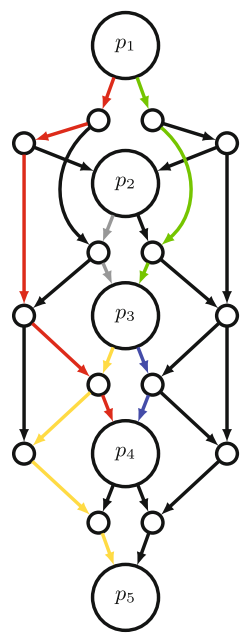

(d) EUG $U_{5}\left(\Gamma_{2}\right)$

Fig. 1. (a) shows an example $\Gamma_{2}(5)$ graph $G$. (b)-(c) show the edge-embedding of $G$ into two $U_{5}\left(\Gamma_{1}\right)$ instances with poles $\left(p_{1}, \ldots, p_{5}\right)$. (d) shows the edge-embedding of $G$ into one $U_{5}\left(\Gamma_{2}\right)$ graph.

EUG $U_{n}\left(\Gamma_{d}\right)$ for $d \geq 2$ can be created by taking $d$ instances of $U_{n}\left(\Gamma_{1}\right)$ EUGs, and merging each pole $p_{i}$ with its multiple instances, allowing the poles to have fanin-fanout $d$. Let $U_{n}\left(\Gamma_{d}\right)=\left(V_{U}^{\prime}, E_{U}^{\prime}\right)$ be an EUG with fanin and fanout $d$, with $U_{n}\left(\Gamma_{1}\right)_{1}=\left(V_{1}, E_{1}\right), \ldots, U_{n}\left(\Gamma_{1}\right)_{d}=\left(V_{d}, E_{d}\right) . P$ contains the merged poles and $V_{U}^{\prime}=P \cup_{i=1}^{d} V_{i} \backslash P_{i}$ and $E_{U}^{\prime}=\cup_{i=1}^{d} E_{i}$.

We give an example for better understanding. Let $G=(V, E)$ be the graph with 5 nodes in Fig. 1a. Our aim is to edge-embed $G$ into EUG $U_{5}\left(\Gamma_{2}\right)$. Therefore, we use two instances of $U_{5}\left(\Gamma_{1}\right): U_{5}\left(\Gamma_{1}\right)_{1}$ in Fig. 1b and $U_{5}\left(\Gamma_{1}\right)_{2}$ in Fig. 1c. The edges $\left(a_{1}, a_{4}\right),\left(a_{2}, a_{3}\right)$ and $\left(a_{3}, a_{5}\right)$ are embedded in $U_{5}\left(\Gamma_{1}\right)_{1}$, and the edges $\left(a_{1}, a_{3}\right)$ and $\left(a_{3}, a_{4}\right)$ in $U_{5}\left(\Gamma_{1}\right)_{2}$. Merging the poles of $U_{5}\left(\Gamma_{1}\right)_{1}$ and $U_{5}\left(\Gamma_{1}\right)_{2}$ produces $U_{5}\left(\Gamma_{2}\right)$ shown in Fig. 1 d.

\subsection{Valiant's UC Constructions}

The size of a function $f$ represented by a circuit $C_{u, v}^{k}$ with fanin and fanout 2 is $n=u+v+k$. In the following, we describe Valiant's UC construction [Val76, Weg87] that can be programmed to evaluate any function of size $n$. Circuit $C_{u, v}^{k}$ is represented as a graph $G \in \Gamma_{2}(n)$ (cf. Sect. 2.1).

Valiant's UC is based on an EUG $U_{n}\left(\Gamma_{2}\right)=\left(V_{U}, E_{U}\right)$ with fanin and fanout 2, which can be transformed to a Boolean circuit. $P \subseteq V_{U}$ contains the poles of $U_{n}\left(\Gamma_{2}\right)$ (cf. Sect. 2.1). Poles $\{1, \ldots, u\}$ correspond to the inputs, $\{(u+$ $1), \ldots,(u+k)\}$ to the gates, $\{(u+k+1), \ldots, n\}$ to the outputs of $C_{u, v}^{k}$. The edges of the graph of the circuit $G=(V, E)$ have to be embedded into $U_{n}\left(\Gamma_{2}\right)$. After the transformations described in Sect. 2.1, every node in $G$ has fanin and 
fanout 2 , and we denote a topological order on $V$ by $\eta^{G}$. We briefly describe the edge-embedding process in Sects. 2.3 and 3.

Translating a $\boldsymbol{U}_{\boldsymbol{n}}\left(\boldsymbol{\Gamma}_{\mathbf{2}}\right)$ into a Universal Circuit. Every node $w \in V_{U}$ fulfills a task when $U_{n}\left(\Gamma_{2}\right)$ is translated to a UC. Programming the UC means specifying its control bits along the paths defined by the edge-embedding and by the gates of circuit $C_{u, v}^{k}$. Depending on the number of incoming and outgoing edges and its type, a node is translated to:

G1. If $w$ is a pole and corresponds to an input or output in $G$, then $w$ is an input or output in $U_{n}\left(\Gamma_{2}\right)$ as well.

G2. If $w$ is a pole and corresponds to a gate in $G, w$ is programmed as a universal gate (UG). A 2-input UG supports any of the 16 possible gate types represented by the 4 control bits of the gate table $\left(c_{1}, c_{2}, c_{3}, c_{4}\right)$. It implements function $u g$ : $\{0,1\}^{2} \times\{0,1\}^{4} \rightarrow\{0,1\}$ that computes:

$$
u g\left(x_{1}, x_{2}, c_{1}, c_{2}, c_{3}, c_{4}\right)=\overline{x_{1} x_{2}} c_{1}+\overline{x_{1}} x_{2} c_{2}+x_{1} \overline{x_{2}} c_{3}+x_{1} x_{2} c_{4} .
$$

Generally, a UG can be implemented with 3 AND and 6 XOR gates (resp. with a two-input gate when using Yao's protocol for SFE) [KS16].

G3. If $w$ is no pole and has indegree and outdegree 2, $w$ is programmed as an $X$-switching block, that computes $f_{X}:\{0,1\}^{2} \times\{0,1\} \rightarrow\{0,1\}^{2}$ with $f_{X}\left(\left(x_{1}, x_{2}\right), c\right)=\left(x_{1+c}, x_{2-c}\right)$. This block can be implemented with 1 AND and 3 XORs (resp. a one-input gate with Yao) [KS08a].

G4. If $w$ is no pole and has indegree 2 and outdegree $1, w$ is programmed as a $Y$-switching block that computes $f_{Y}:\{0,1\}^{2} \times\{0,1\} \rightarrow\{0,1\}$ with $f_{Y}\left(\left(x_{1}, x_{2}\right), c\right)=x_{1+c}$. This block can be implemented with 1 AND and 2 XORs (resp. a one-input gate with Yao) [KS08a].

G5. If $w$ is no pole and has indegree 1 and outdegree 2 , it has been placed to copy its input to its two outputs. Therefore, when translated to a UC, $w$ is replaced by multiple outgoing wires in the parent node [KS16], since the UC itself does not have the fanout 2 restriction. In $U_{n}\left(\Gamma_{2}\right), w$ is added due to the fanout 2 restriction in the EUG.

G6. If $w$ is no pole and has indegree and outdegree $1, w$ is removed and replaced by a wire between its parent and child nodes.

The nodes programmed as UG (G2), X-switching block (G3) or Y-switching block (G4) are so-called programmable blocks. This means that a programming bit or vector is necessary besides the two inputs to define their behavior as described above. These programming bits and vectors that build up the programming of the UC $p_{f}$ are defined by the paths in the edge-embedding of $G$ (the graph of circuit $C_{u, v}^{k}$ describing $f$ ) into $U_{n}\left(\Gamma_{2}\right)$.

Recursion Base. Valiant's construction is recursive, and the recursion base is reached when the number of poles is between 1 and 6 . These recursion base graphs are shown in [Val76, KS16]. $U_{1}\left(\Gamma_{1}\right)$ is a single pole, $U_{2}\left(\Gamma_{1}\right)$ and $U_{3}\left(\Gamma_{1}\right)$ are two and three connected poles, respectively. $U_{4}\left(\Gamma_{1}\right), U_{5}\left(\Gamma_{1}\right)$ and $U_{6}\left(\Gamma_{1}\right)$ are constructed with 3,7 and 9 additional nodes, respectively. 


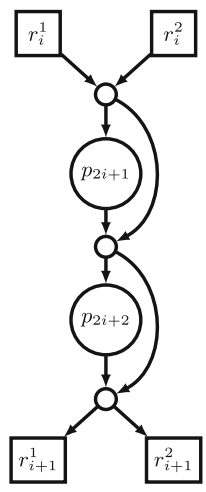

(a) Body Block (2)

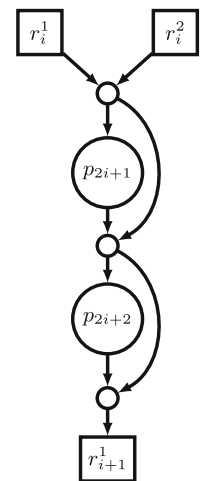

(b) Body Block (1)

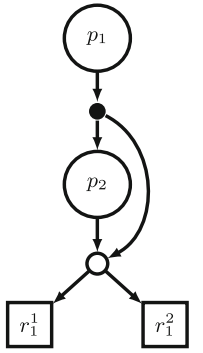

(c) Head Block

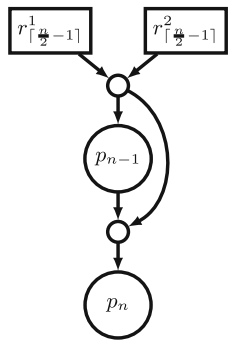

(d) Tail Block (2)

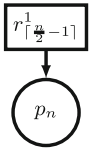

(e) Tail Block (1)

Fig. 2. (a) shows Valiant's 2-way EUG $U_{n}^{(2)}\left(\Gamma_{1}\right)$ [Val76]. (c) shows the corresponding head block, (b) and (d)-(e) show body and tail blocks, respectively, for different numbers of poles.

\subsection{Valiant's 2-Way UC Construction}

We described in Sect. 2.1 that a $U_{n}\left(\Gamma_{d}\right)$ EUG can be constructed of $d$ instances of $U_{n}\left(\Gamma_{1}\right)$ EUGs. Therefore, Valiant provides an EUG for $\Gamma_{1}(n)$ graphs, two of which can build an EUG for $\Gamma_{2}(n)$ graphs. Let $P=\left\{p_{1}, \ldots, p_{n}\right\}$ be the set of poles in $U_{n}^{(2)}\left(\Gamma_{1}\right)$ that have indegree and outdegree 1. Valiant's 2-way EUG construction for $\Gamma_{1}(n)$ graphs of size $\sim 2.5 n \log _{2} n$ is shown in Fig. 2, where we emphasize the poles as large circles and the additional nodes as small circles or rectangles. The corresponding UC has twice the size $\sim 5 n \log _{2} n$, since it corresponds to the EUG for $\Gamma_{2}(n)$ graphs.

The rectangles are special nodes that build the set of poles in the next recursion step, i.e., $R_{\left\lceil\frac{n}{2}-1\right\rceil}^{1}=\left\{r_{1}^{1}, \ldots, r_{\left\lceil\frac{n}{2}-1\right\rceil}^{1}\right\}, R_{\left\lceil\frac{n}{2}-1\right\rceil}^{2}=\left\{r_{1}^{2}, \ldots r_{\left\lceil\frac{n}{2}-1\right\rceil}^{2}\right\}$. Another EUG is built with these poles which produces new subgraphs with size $\left\lceil\frac{\left\lceil\frac{n}{2}-1\right\rceil}{2}-1\right\rceil$, s.t. we have four subgraphs at this level.

This construction is called the 2-way EUG or UC construction. An open-source implementation of this construction optimized for PFE is provided in [KS16]. Independently, [LMS16] also implemented this 2-way UC, additionally optimizing for the total number of gates.

\subsection{Valiant's 4-Way UC Construction}

Valiant provides another, so-called 4-way EUG or UC construction [Val76]. $U_{n}^{(4)}\left(\Gamma_{1}\right)$ has a 4 -way recursive structure, i.e., nodes in special sets $R_{\left\lceil\frac{n}{4}-1\right\rceil}^{1}$, $R_{\left\lceil\frac{n}{4}-1\right\rceil}^{2}, R_{\left\lceil\frac{n}{4}-1\right\rceil}^{3}$ and $R_{\left\lceil\frac{n}{4}-1\right\rceil}^{4}$ are the poles in the next recursion step (cf. Fig. 4a). The recursion base is the same as in Sect. 2.2. This construction results in UCs of 


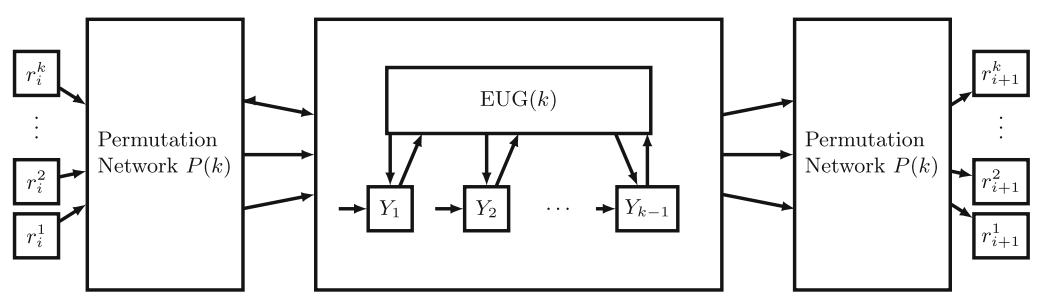

Fig. 3. $k$-way EUG construction $U_{n}^{(k)}\left(\Gamma_{1}\right)$ [LMS16].

smaller size $\sim 4.75 n \log _{2} n$ but has not been implemented before due to its more complicated structure.

\subsection{Lipmaa et al.'s Generalized $k$-Way UC Construction}

In [LMS16], Lipmaa et al. generalize Valiant's approach by providing a UC with any number of recursion points $k$, the so-called $k$-way EUG or UC construction. We note that their construction slightly differs from Valiant's EUG construction, since they do not consider the restriction on the fanout of the poles, i.e., the nodes in the EUG that correspond to universal gates or inputs (cf. Sect. 2.2). This optimization has also been included in [KS16] when translating an EUG to a UC, but including it in the block design leads to better sizes for the number of XOR gates.

The idea is to split $n=u+v+k$ in $m=\left\lceil\frac{n}{k}\right\rceil$ blocks as shown in Fig. 3. Every block $i$ consists of $k$ inputs $r_{i}^{1}, r_{i}^{2}, \ldots, r_{i}^{k}$ and $k$ outputs $r_{i+1}^{1}, r_{i+1}^{2}, \ldots, r_{i+1}^{k}$ as well as $k$ poles, except for the last block which has a number of poles depending on $n \bmod k$. For every $j \leq k$, the list of all $r_{i}^{j}$ builds the poles of the $\mathrm{j}^{\text {th }}$ subgraph of the next recursion step, i.e. we have $k$ subgraphs. Additionally, every block begins and ends with a Waksman permutation network [Wak68] such that the inputs and outputs can be permuted to every pole. A Y-switching block is placed in front of every pole $p_{i}$ which is connected to the $\mathrm{i}^{\text {th }}$ output of the permutation network as well as the $\mathrm{i}^{\text {th }}$ output of a block-intern EUG $U_{k}\left(\Gamma_{1}\right)$. Thus, [LMS16] reduce the problem of finding an efficient $k$-way EUG $U_{n}^{(k)}\left(\Gamma_{2}\right)$ to the problem of finding the smallest EUG $U_{k}\left(\Gamma_{1}\right)$. Their solution is to build the block-intern EUG with the UC construction of [KS08b], which was claimed to be more efficient for smaller circuits than [Val76]. However, they calculate the optimal $k$ value to be around 3.147, which implies that the best solutions are found using small EUGs, for which Valiant provides hand-optimized solutions (i.e., for $k=2,3,4,5,6)$ [Val76].

\section{Our Modular Edge-Embedding Algorithm}

The detailed embedding algorithm and the open-source UC implementation of [KS16] was specifically built for the 2 -way UC, dealing with the whole UC skeleton as one block. In contrast, based on the modular design of [LMS16], we modularize the edge-embedding task into multiple sub-tasks and describe how they 
can be performed separately. In this section, we detail this modular approach for edge-embedding a graph into Valiant's 4-way EUG: the edge-embedding can be split into two parts, which are then combined. In Sect.3.1, we describe our modular approach based on the edge-embedding algorithm of [KS16] for Valiant's 2way EUG. This can be generalized to any $2^{i}$-way EUG construction. Moreover, the same algorithm can be applied with a few modifications for Lipmaa et al.'s $k$-way recursive generalization [LMS16], which we describe in Sect. 3.2.

\subsection{Edge-Embedding in Valiant's 4-Way UC}

Similar to the 2-way EUG construction (cf. Sect.2.3), Valiant provides a 4-way EUG construction for $\Gamma_{1}(n)$ graphs which can be extended to an EUG for $\Gamma_{2}(n)$ graphs by utilizing two instances $U_{n}^{(4)}\left(\Gamma_{1}\right)_{1}$ and $U_{n}^{(4)}\left(\Gamma_{1}\right)_{2}$ as described in Sect. 2.1. The construction with our optimizations is visualized in Fig. 4. Valiant offers the main, so-called Body Block (Fig. 4a) consisting of 4 poles (large circles), 15 nodes (small circles) as well as 8 recursion points (squares). These body blocks are connected such that the 4 top [bottom] recursion points of one block are the 4 bottom [top] recursion points of the next block. Similarly to the 2-way EUG, 4 sets are created for $n$ nodes, i.e., $R_{\left\lceil\frac{n}{4}-1\right\rceil}^{1}=\left\{r_{1}^{1}, \ldots, r_{\left\lceil\frac{n}{4}-1\right\rceil}^{1}\right\}, R_{\left\lceil\frac{n}{4}-1\right\rceil}^{2}=\left\{r_{1}^{2}, \ldots, r_{\left\lceil\frac{n}{4}-1\right\rceil}^{2}\right\}$, $R_{\left\lceil\frac{n}{4}-1\right\rceil}^{3}=\left\{r_{1}^{3}, \ldots, r_{\left\lceil\frac{n}{4}-1\right\rceil}^{3}\right\}$, and $R_{\left\lceil\frac{n}{4}-1\right\rceil}^{4}=\left\{r_{1}^{4}, \ldots, r_{\left\lceil\frac{n}{4}-1\right\rceil}^{4}\right\}$ which are the poles of $4 U_{\left\lceil\frac{n}{2}\right\rceil-1}\left(\Gamma_{1}\right)$ EUGs in the next recursion step. Then, these also create 4 subgraphs until the recursion base is reached, cf. Sect. 2.2.

We note that the top [bottom] block does not need the upper [lower] recursion points since its poles are the inputs [outputs] in the block. Therefore, we provide so-called Head and Tail Blocks. A Head Block occurs at the top of a chain of blocks (cf. Fig. 4e), it has 4 poles, no inputs, 4 output recursion points and 10 nodes, of which the first one (denoted by a filled circle) has one input and therefore translates to wires in the UC.

As a counterpart, Tail Blocks occur at the bottom of a chain of blocks, have at most 4 poles, 4 input recursion points, no outputs and at most 10 nodes depending on the number of poles. The 4 tail block constructions are depicted in Figs. $4 \mathrm{f}-\mathrm{i}$ and are used, based on the remainder of $n$ modulo 4 , with the respective body or head blocks when $n \in\{5,6,7\}$, the lower parts of which are shown in Figs. 4a-d.

Block Edge-Embedding. In this first part of the edge-embedding process, we consider the 4 top [bottom] recursion points of the block as intermediate nodes where the inputs [outputs] of the block enter [leave]. The blocks are built s.t. any of these inputs can be forwarded to exactly one of the 4 poles of the block and the output of any pole can be forwarded to exactly one output or another pole having a higher topological order.

We formalize this behaviour as follows: In $U_{n}^{(4)}\left(\Gamma_{1}\right)=\left(V_{U}, E_{U}\right)$, let $B$ be the block visualized in Fig. 4a with poles $p_{4 i+1}, \ldots, p_{4 i+4}$. Let mapping $\eta^{U}: V_{U} \rightarrow \mathbb{N}^{+}$ denote a topological order of $V_{U}$. Then, the nodes $r_{i}^{1}, \ldots, r_{i}^{4}$ and $r_{i+1}^{1}, \ldots, r_{i+1}^{4}$ denote the input and output recursion points of block $B$. Additionally, let in = 


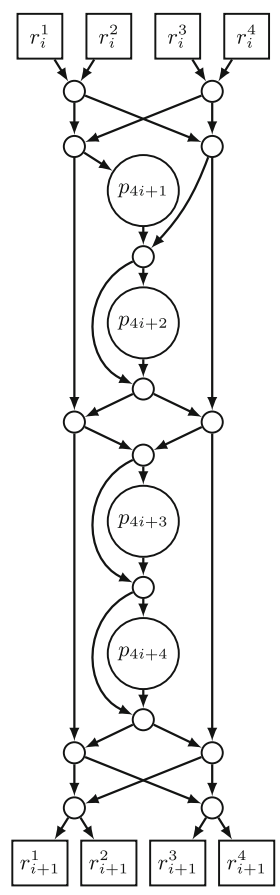

(a) Body Block (4)

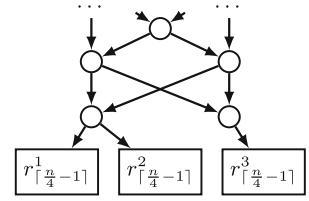

(b) Body/Head Block (3)

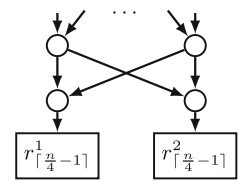

(c) Body/Head Block (2)

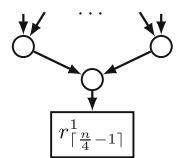

(d) Body/Head Block (1)

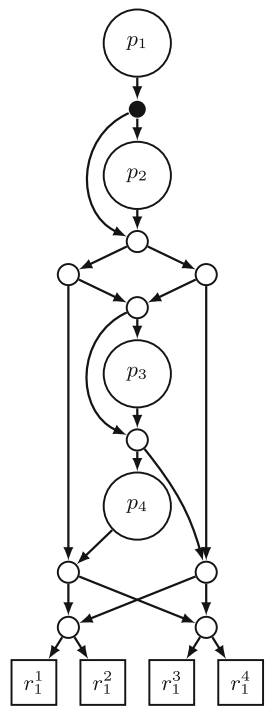

(e) Head Block (4)

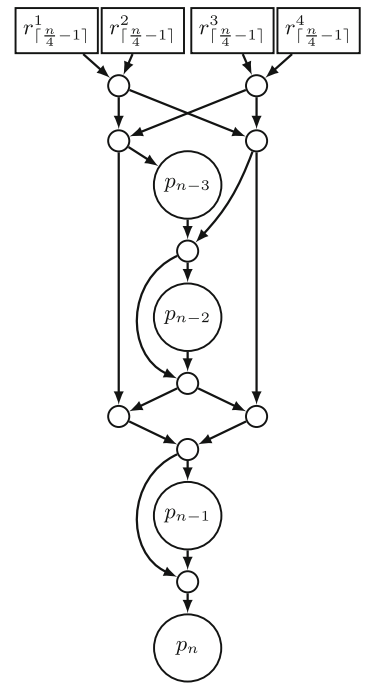

(f) Tail Block (4)

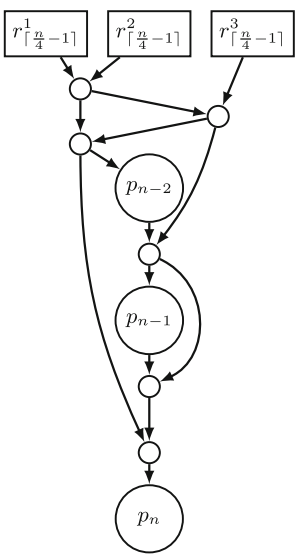

(g) Tail Block (3)

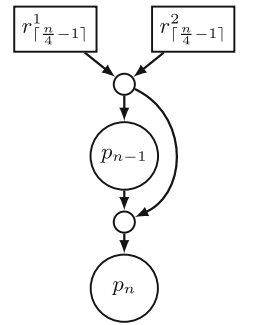

(h) Tail Block (2)

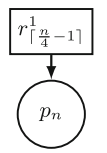

(i) Tail Block (1)

Fig. 4. (a) shows Valiant's 4-way EUG $U_{n}^{(4)}\left(\Gamma_{1}\right)$ [Val76]. (e) shows our head block construction, (a)-(d) and (f)-(i) show our body and tail block constructions, respectively, for different numbers of poles. 
$\left(\right.$ in $_{1}, \ldots$, in $\left._{4}\right) \in\{0, \ldots, 4\}^{4}$ and out $=\left(\right.$ out $_{1}, \ldots$, out $\left._{4}\right) \in\{0, \ldots, 7\}^{4}$ denote the input and output vectors of $B$. The value 0 of the input and output vectors is a dummy value which is used if an input [a pole] is not forwarded to any pole [output] of $B$. The output vector has a larger value range, since a pole can be forwarded to another pole or an output recursion point. Therefore, we use values 1, 2 and 3 for poles $p_{2}, p_{3}$ and $p_{4}$ and values $4,5,6$ and 7 for the output recursion points. Pole $p_{1}$ cannot be a destination for a path in $B$, since $\eta^{U}\left(p_{1}\right)$ is less than the topological order of any other pole in $B$. Additionally, the values of in and out need to be pairwise different or 0 . Every combination of input and output vector covering the conditions formalized below in Eqs. 2-6 are valid for $B$. A pair $\left(r_{i}^{l}, p_{j}\right) \in \mathcal{P}$ or $\left(p_{j}, r_{i+1}^{l}\right) \in \mathcal{P}$ is a path from $r_{i}^{l}$ to $p_{j}$ or $p_{j}$ to $r_{i}^{l}$ in the set of all paths $\mathcal{P}$ in $B$. Then, $\mathcal{P}_{B} \subseteq \mathcal{P}$ denote the paths that are to be edge-embedded (cf. Sect. 6.1).

$$
\begin{aligned}
& \forall l \in\{1, \ldots, 4\}: \text { in }_{l} \neq 0 \rightarrow\left(r_{i}^{l}, p_{\text {in }_{l}}\right) \in \mathcal{P}_{B}, \\
& \text { out }_{l} \neq 0 \wedge \text { out }_{l}<4 \rightarrow\left(p_{j}, p_{1+\text { out }_{l}}\right) \in \mathcal{P}_{B} \wedge \eta^{U}\left(p_{j}\right)<\eta^{U}\left(p_{1+\text { out }_{l}}\right) \\
& \qquad \text { out }_{l}>3 \rightarrow\left(p_{j}, r_{i+1}^{l-3}\right) \in \mathcal{P}_{B} . \\
& \forall \text { in }_{i}, \text { in }_{j} \in \text { in }: i \neq j \rightarrow \text { in }_{i}=0 \vee \text { in }_{i} \neq i n_{j} . \\
& \forall \text { out }_{i}, \text { out }_{j} \in \text { out }: i \neq j \rightarrow \text { out }_{i}=0 \vee \text { out }_{i} \neq \text { out }_{j} .
\end{aligned}
$$

Recursion Point Edge-Embedding. The block edge-embedding covers only the programming of the nodes within a block. Another task left is to program the recursion points. We use the supergraph construction of [KS16] which, in every step, splits a $\Gamma_{2}(n)$ graph in two $\Gamma_{1}(n)$ graphs, which are merged to two $\Gamma_{2}\left(\left\lceil\frac{n}{2}-1\right\rceil\right)$ graphs. [KS16] use this for defining the paths in Valiant's 2-way EUG. For Valiant's 4-way EUG, we use every second step of their algorithm with a minor modification.

Let $C_{u, v}^{k}$ be the Boolean circuit computing function $f$ that our UC needs to compute, and $G \in \Gamma_{2}(n)$ its graph representation (cf. Sect.2.2).

1. Splitting $G \in \Gamma_{2}(n)$ in two $\Gamma_{1}(n)$ graphs $G_{1}$ and $G_{2}$ : As described in Sect. 2.1, Valiant's UC is derived from an EUG for $\Gamma_{2}(n)$ graphs, which consists of two EUGs for $\Gamma_{1}(n)$ graphs merged by their poles. Therefore, $G$ is split into two $\Gamma_{1}(n)$ graphs $G_{1}$ and $G_{2} . G_{1}$ and $G_{2}$ then need to be edge-embedded into EUGs $\left(U_{n}^{(4)}\left(\Gamma_{1}\right)\right)_{1}$ and $\left(U_{n}^{(4)}\left(\Gamma_{1}\right)\right)_{2}$, respectively. $G=(V, E) \in \Gamma_{2}(n)$ is split by 2-coloring its edges as described in [Val76, KS16], which can always be done due to König's theorem [Kö31,LP09]. After 2-coloring, $E$ is divided to sets $E_{1}$ and $E_{2}$, using which we build $G_{1}=\left(V, E_{1}\right)$ and $G_{2}=\left(V, E_{2}\right)$, with the following conditions:

$$
\begin{array}{r}
\forall e \in E:\left(e \in E_{1} \vee e \in E_{2}\right) \wedge \neg\left(e \in E_{1} \wedge e \in E_{2}\right) . \\
\forall e=\left(v_{1}, v_{2}\right) \in E_{1}: \neg \exists e^{\prime}=\left(v_{3}, v_{4}\right) \in E_{1}: v_{2}=v_{4} \vee v_{1}=v_{3} . \\
\forall e=\left(v_{1}, v_{2}\right) \in E_{2}: \neg \exists e^{\prime}=\left(v_{3}, v_{4}\right) \in E_{2}: v_{2}=v_{4} \vee v_{1}=v_{3} .
\end{array}
$$

2. Merging a $\Gamma_{1}(n)$ graph into a $\Gamma_{2}\left(\left\lceil\frac{n}{2}-1\right\rceil\right)$ graph: In an EUG, the number of poles decreases in each recursion step and therefore, merging a $\Gamma_{1}(n)$ 
graph into a $\Gamma_{2}\left(\left\lceil\frac{n}{2}-1\right\rceil\right)$ graph provides information about the paths to be taken. Let $G_{1}=(V, E) \in \Gamma_{1}(n)$ be a topologically ordered graph and $G_{m}=\left(V^{\prime}, E^{\prime}\right) \in \Gamma_{2}\left(\left\lceil\frac{n}{2}\right\rceil\right)$ be a graph with nodes $v_{1}^{\prime}, \ldots, v_{\left\lceil\frac{n}{2}\right\rceil}^{\prime}$. We define two labellings $\eta_{\text {in }}$ and $\eta_{\text {out }}$ on $G_{m}$ with $\eta_{\text {in }}\left(v_{i}\right)=i$ and $\eta_{\text {out }}\left(v_{i}\right)=\eta_{\text {in }}\left(v_{i}\right)-1=$ $i-1$. Additionally, we define a mapping $\theta_{V}$ that maps a node $v_{i} \in V$ to a node $v_{j} \in V^{\prime}$ with $\theta_{V}\left(v_{i}\right)=v_{\left\lceil\frac{i}{2}\right\rceil}^{\prime}$. That means two nodes in $G_{1}$ are mapped to one node in $G_{m}$. At last, we define a mapping $\theta_{E}$ that maps an edge $e_{i}=$ $\left(v_{i}, v_{j}\right) \in E$ to an edge $e_{j} \in E^{\prime}$ with $\theta_{E}\left(\left(v_{i}, v_{j}\right)\right)=\left(v_{\eta_{\text {in }}\left(\theta_{V}\left(v_{i}\right)\right)}, v_{\eta_{\text {out }}\left(\theta_{V}\left(v_{j}\right)\right)}\right)$. That means every edge in $G_{1}$ is mapped to an edge in $G_{m}$ as follows: $e=$ $\left(v_{i}, v_{j}\right) \in E$ is mapped to $e^{\prime}=\left(v_{k}^{\prime}, v_{l}^{\prime}\right) \in E^{\prime}$, s.t. $v_{k}^{\prime}=\theta_{V}\left(v_{i}\right)$, but $v_{l}^{\prime}$ is not the new node of $v_{j}$ in $G_{m}$ but $v_{l+1}^{\prime} . G_{m}$ is built as follows: $V^{\prime}=\left\{v_{1}^{\prime}, \ldots, v_{\left\lceil\frac{n}{2}\right\rceil}^{\prime}\right\}$ and $E^{\prime}=\bigcup_{e \in E} \theta_{E}(e)$. Then for all $e=\left(v_{i}^{\prime}, v_{j}^{\prime}\right) \in E^{\prime}$ and $j<i, e$ is removed from $E^{\prime}$, along with the last node $v_{\left\lceil\frac{n}{2}\right\rceil}$ (due to the definition of $\theta_{E}$, it does not have any incoming edges). The resulting $G_{m}$ is a topologically ordered graph in $\Gamma_{2}\left(\left\lceil\frac{n}{2}-1\right\rceil\right)$.

3. The supergraph for Valiant's 4-way EUG construction: In the first step, $G$ is split to two $\Gamma_{1}(n)$ graphs $G_{1}$ and $G_{2} . G_{1}$ and $G_{2}$ contain all the edges that should be embedded as paths between poles in the first and second EUGs for $\Gamma_{1}(n)$, respectively. We now explain how to edge-embed the $\Gamma_{1}(n)$ graph $G_{1}$ into an EUG $U_{n}^{(4)}\left(\Gamma_{1}\right)$ (for $G_{2}$ it is the same).

For embedding in a 2-way UC, $G_{1}$ is firstly merged to a $\Gamma_{2}\left(\left\lceil\frac{n}{2}\right\rceil\right)$ graph $G_{m} . G_{m}$ is then 2-colored and split into two $\Gamma_{1}\left(\left\lceil\frac{n}{2}\right\rceil\right)$ graphs $G_{1}^{1}$ and $G_{1}^{2}[\mathrm{KS} 16]$. These get merged to two $\Gamma_{2}\left(\left\lceil\frac{\left\lceil\frac{n}{2}-1\right\rceil}{2}-1\right\rceil\right)$ graphs $G_{m}^{1}$ and $G_{m}^{2}$. $G_{1}^{1}$ is the first and $G_{1}^{2}$ is the second subgraph of $G_{1}$. Then $G_{1}^{\psi \circ 1}$ and $G_{1}^{\psi \circ 2}$ denote the first and second subgraph of $G_{1}^{\psi}$, respectively. These steps are repeated until the $\Gamma_{1}$ subgraphs have at most 4 nodes.

In Valiant's 4-way EUG construction [Val76], a supergraph that creates 4 subgraphs in each step is necessary. We require a merging method where a $\Gamma_{1}(n)$ graph is merged to a $\Gamma_{4}\left(\left\lceil\frac{n}{4}-1\right\rceil\right)$ graph where 4 nodes build a new node, and 4 -color this graph to retrieve 4 subgraphs. However, this can directly be solved by using the method described above from [KS16]: after repeating the 2-coloring and the merging twice, we gain 4 subgraphs $\left(G_{1}^{11}, G_{1}^{12}, G_{1}^{21}\right.$ and $\left.G_{1}^{22}\right)$. These can be used as if they were the result of 4 -coloring the graph obtained by merging every 4 nodes into one.

However, there is a modification in this case: the first 2-coloring is a preprocessing step, which does not map to an EUG recursion step. Therefore, we have to define another labelling $\eta_{\text {out }_{P}}(v)=\eta_{\text {in }}(v)$, since in this preprocessing step we need to keep node $v_{\left\lceil\frac{n}{2}\right\rceil}$. Then the creation of the supergraph for the 4way EUG construction works as follows: We merge $G_{1}$ to a $\Gamma_{2}\left(\left\lceil\frac{n}{2}\right\rceil\right)$ graph with labelling $\eta_{\text {in }}$ and $\eta_{\text {out }_{P}}$ and get $G_{m}$. After that, we split $G_{m}$ into two $\Gamma_{1}\left(\left\lceil\frac{n}{2}\right\rceil\right)$ graphs $G_{1}^{1}$ and $G_{1}^{2}$. These get merged to $\Gamma_{2}\left(\left\lceil\frac{n}{4}\right\rceil-1\right)$ graphs $G_{m}^{1}$ and $G_{m}^{2}$ using the $\eta_{\text {in }}$ and $\eta_{\text {out }}$ labellings. Finally, these two graphs get splitted into $4 \Gamma_{1}\left(\left\lceil\frac{n}{4}-1\right\rceil\right)$ graphs $G_{1}^{11}, G_{1}^{12}, G_{1}^{21}$ and $G_{1}^{22}$. These are the relevant graphs for the first recursion 
Listing 1. Edge-embedding algorithm for Valiant's 4-way EUG

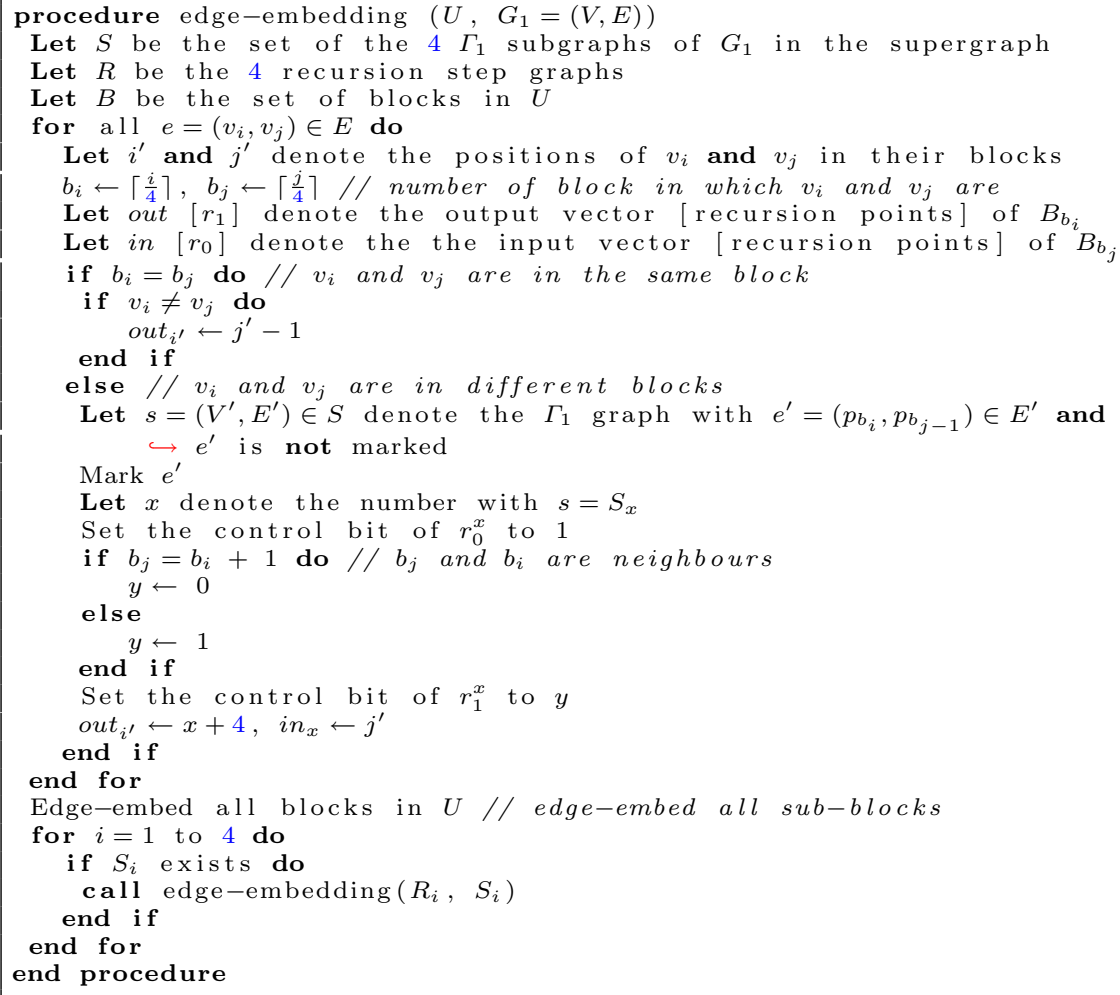

step in Valiant's 4-way EUG construction. Now we continue for all 4 subgraphs until we reach the recursion base with 4 or less nodes.

4-Way Edge-Embedding Algorithm. In Listing 1, we combine block edgeembedding and recursion point edge-embedding:

Let $U$ denote the part of $U_{n}^{(4)}\left(\Gamma_{1}\right)$ without recursion steps (the main chain of blocks) and $G_{1}=(V, E)$ be the $\Gamma_{1}(n)$ graph which is to be edge-embedded in $U_{n}^{(4)}\left(\Gamma_{1}\right) . S$ denotes the set of the 4 subgraphs of $G_{1}$ in the supergraph, i.e. $S=$ $\left\{G_{1}^{11}, G_{1}^{12}, G_{1}^{21}, G_{1}^{22}\right\}$. A recursion step graph of $U$ is one of the graphs having one of the 4 sets of recursion points as poles (e.g. $r_{1}^{1}, \ldots, r_{\left\lceil\frac{n}{4}-1\right\rceil}^{1}$ ) without the recursion steps. $R$ denotes the set of all 4 recursion step graphs of $U$, and $B$ denotes the set of all blocks in $U$.

We give a brief explanation of Listing 1 that describes the edge-embedding process. For any edge $e=\left(v_{i}, v_{j}\right) \in E$ in $G_{1}, b_{i}$ and $b_{j}$ denote the block numbers in which $v_{i}$ and $v_{j}$ are. There are 2 cases: 
1. $v_{i}$ and $v_{j}$ are in the same block: $b_{i}=b_{j}$. The edge-embedding can be solved within the block and no recursion points have to be programmed for this path. Therefore, vector out of block $B_{b_{i}}$ is set accordingly.

2. $v_{i}$ and $v_{j}$ are in different blocks: $b_{i} \neq b_{j}$. There exists an edge $e^{\prime}=\left(b_{i}, b_{j-1}\right)$ in one of the four $\Gamma_{1}\left(\left\lceil\frac{n}{4}-1\right\rceil\right)$ subgraphs of $G_{1}$ that is not yet used for an edgeembedding. This determines that the path in the next recursion step has to be between poles $p_{b_{i}}$ and $p_{b_{j-1}}$. We denote with $s \in S$ the subgraph of $G_{1}$ which contains $e^{\prime}$, and $x$ denotes its number in $S$, i.e. $S_{x}=s$. This implies in which of the 4 recursion step graphs we need to edge-embed the path from $p_{b_{i}}$ to $p_{b_{j-1}}$, and so which recursion points we need to program. We first set the programming bit of the $x$-th input [output] recursion points to 1 since the path between the poles with labelling $i$ and $j$ enters [leaves] the next recursion step over this recursion point. A special case to be considered here is when blocks $B_{b_{i}}$ and $B_{b_{j}}$ are neighbours (i.e. $b_{j}=b_{i}+1$ ). Then, the path enters and leaves the next recursion step graph at the same node, whose programming bit thus has to be 0 . The output vector of block $B_{b_{i}}$ is the $i^{\text {th }}$ value to the $x^{\text {th }}$ recursion point and the input vector of block $B_{b_{j}}$ is the $x^{\text {th }}$ value to the $j^{\text {th }}$ pole in this block.

We repeat these steps for all edges $e \in E$. Since all in- and output vectors of all blocks in $B$ are set, they can be embedded with the block edge-embedding. For all 4 subgraphs of $G_{1}$ in the supergraph and in the EUG, we call the same procedure with $S_{i} \in S, R_{i} \in R, 1 \leq i \leq 4$.

\subsection{Edge-Embedding in Lipmaa et al.'s $k$-Way UC}

In this section, we extend the recent work of [LMS16] by providing a detailed and modular embedding mechanism for any $k$-way EUG construction described in Sect. 2.5. We provide the main differences to the edge-embedding of the 4-way EUG construction detailed in Sect. 3.1.

$\boldsymbol{k}$-Way Block Edge-Embedding. In this setting, our main block is a programmable block $B$ of size $x$ with $k$ poles $p_{1}, \ldots, p_{k}$, and $k$ input [output] recursion points $r_{0}^{1}, \ldots, r_{0}^{k}\left[r_{1}^{1}, \ldots, r_{1}^{k}\right] . B$ is topologically ordered with mapping $\eta^{U}$ as defined in Sect. 2.1. Vectors $i n=\left(i n_{1}, \ldots, i n_{k}\right) \in\{0, \ldots, k\}^{k}$, and out $=\left(\right.$ out $_{1}, \ldots$, out $\left._{k}\right) \in\{0, \ldots, 2 k-1\}^{k}$ denote the input and output vectors of $B$, respectively. Values $k, \ldots, 2 k-1$ in out denote the recursion point targets $r_{1}^{1}, \ldots, r_{1}^{k}$ (cf. Sect. 3.1). We formalize the setting of $i n$ and out in Eqs. 10-14. We denote with $\mathcal{P}$ the set of all paths in $B$, and the $\mathcal{P}_{B} \subseteq P$ the paths that get edge-embedded in $B$.

$$
\begin{aligned}
& \forall i \in\{1, \ldots, k\}: i n_{i} \neq 0 \rightarrow\left(r_{0}^{i}, p_{i n_{i}}\right) \in \mathcal{P}_{B}, \\
& \text { out }_{i} \neq 0 \wedge \text { out }_{i}<k \rightarrow\left(p_{i}, p_{1+\text { out }_{i}}\right) \in \mathcal{P}_{B} \wedge \eta^{U}\left(p_{i}\right)<\eta^{U}\left(p_{1+\text { out }_{i}}\right) \\
& \text { out }_{i}>k-1 \rightarrow\left(p_{i}, r_{1}^{i-k+1}\right) \in \mathcal{P}_{B} . \\
& \forall i n_{i}, i n_{j} \in i n: i \neq j \rightarrow i n_{i}=0 \vee i n_{i} \neq i n_{j} . \\
& \text { out }_{i}, \text { out }_{j} \in \text { out }: i \neq j \rightarrow \text { out }_{i}=0 \vee \text { out }_{i} \neq \text { out }_{j} \text {. }
\end{aligned}
$$


$\boldsymbol{k}$-Way Recursion Point Edge-Embedding. $G \in \Gamma_{2}(n)$ denotes the transformed graph of a Boolean circuit $C_{u, v}^{k}$, where $n=u+k+v$.

1. Splitting $G \in \Gamma_{2}(n)$ in two $\Gamma_{1}(n)$ graphs $G_{1}$ and $G_{2}$ : Similarly as in Sect. 3.1, we first split $G$ into two $\Gamma_{1}(n)$ graphs $G_{1}$ and $G_{2}$ with 2-coloring.

2. Merging a $\Gamma_{1}(n)$ graph into a $\Gamma_{k}\left(\left\lceil\frac{n}{k}-1\right\rceil\right)$ graph: $G_{1}=(V, E) \in \Gamma_{1}(n)$ is merged into a $\Gamma_{k}\left(\left\lceil\frac{n}{k}-1\right\rceil\right)$ graph $G_{m}=\left(V^{\prime}, E^{\prime}\right)$ (same for $\left.G_{2}\right)$. Therefore, we redefine mapping $\theta_{V}$ (cf. Sect. 3.1) that maps node $v_{i} \in V$ to node $v_{j} \in V^{\prime}$. In this scenario, $k$ nodes in $V$ build one node in $V^{\prime}$, so $\theta_{V}\left(v_{i}\right)=v_{\left\lceil\frac{i}{k}\right\rceil}$. The mapping of the edges $\theta_{E}$ is the same as in the 4-way EUG construction, and $\left(v_{i}^{\prime}, v_{j}^{\prime}\right) \in E^{\prime}$ where $j<i$ edges are removed along with $v_{\left\lceil\frac{n}{k}\right\rceil}$ in the end. $G_{m}$ is then a topologically ordered graph in $\Gamma_{1}\left(\left\lceil\frac{n}{k}-1\right\rceil\right)$.

3. The supergraph for Lipmaa et al.'s k-way EUG construction: The next step is to split $G_{m} \in \Gamma_{1}\left(\left\lceil\frac{n}{k}-1\right\rceil\right)$ into $k \Gamma_{1}\left(\left\lceil\frac{n}{k}-1\right\rceil\right)$ graphs. This is done with $k$-coloring: a directed graph $K=(V, E)$ can be $k$-colored, if $k$ sets $E_{1}, \ldots, E_{k} \subseteq$ $E$ cover the following conditions:

$$
\begin{aligned}
& \forall i, j \in\{1, \ldots, k\}: i \neq j \rightarrow E_{i} \cap E_{j}=\emptyset . \\
& \forall e \in E: \exists i \in\{1, \ldots, k\}: e \in E_{i} . \\
& \forall i \in\{1, \ldots, k\}: \forall e=\left(v_{1}, v_{2}\right) \in E_{i}: \\
& \quad \neg \exists e^{\prime}=\left(v_{3}, v_{4}\right) \in E_{i}: v_{2}=v_{4} \vee v_{1}=v_{3} .
\end{aligned}
$$

According to König's theorem [Kő31,LP09], $\Gamma_{k}(n)$ graphs can always be $k$ colored efficiently (cf. full version [GKS17, Appendix A] for details). The rest of the supergraph construction and the way it is used for edge-embedding is the same as for the 4-way EUG construction as described in Sect. 3.1.

$\boldsymbol{k}$-Way Edge Embedding Algorithm. The edge-embedding algorithm is the same as shown in Listing 1 , after replacing every 4 with $k$.

\section{New Universal Circuit Constructions}

Here, we describe our ideas for novel, potentially more efficient, UC constructions. Firstly, in Sect. 4.1, we describe modular building blocks for a 3-way UC. We show that Valiant's optimized $U_{3}\left(\Gamma_{1}\right)$ cannot directly be applied as a building block in the construction due to the fact that it must have an additional node to be a generic EUG. We prove that the EUG without this node is not a valid EUG by showing a counterexample. Therefore, it actually results in a worse asymptotic size than Valiant's 2-way and 4-way UC constructions. Secondly, in Sect. 4.2, we propose a hybrid UC construction, utilizing both Valiant's 2-way and 4-way UC constructions so that the overall size of the resulting hybrid UC is minimized, and is at least as efficient as the better construction for the given size. 


\subsection{3-Way Universal Circuit Construction}

The optimal $k$ value for minimizing the size of the $k$-way UC was calculated to be 3.147 in [LMS16]. We describe our idea of a 3-way UC construction. Intuitively, based on an optimization by Valiant [Val76], this UC should result in the best asymptotic size. The asymptotic size of any $k$-way UC depends on the size of its modular body block $B_{k}$ (e.g., Fig. 4a for the 4-way UC). Once it is determined, the size of the UC is $\operatorname{size}\left(U_{n}^{(k)}\left(\Gamma_{2}\right)\right)=2 \cdot \operatorname{size}\left(U_{n}^{(k)}\left(\Gamma_{1}\right)\right) \approx 2 \cdot \frac{\operatorname{size}\left(B_{k}\right)}{k} n \log _{k} n=$ $2 \cdot \frac{\operatorname{size}\left(B_{k}\right)}{k \log _{2}(k)} n \log _{2} n$. The modular block consists of two permutation networks $P(k)$, an EUG $U_{k}\left(\Gamma_{1}\right)$, and $(k-1)$ Y-switching blocks (cf. Sect. 2.5, [LMS16]).

Size of Body Block $\boldsymbol{B}_{\mathbf{3}}$ with Valiant's Optimized $\boldsymbol{U}_{\mathbf{3}}\left(\boldsymbol{\Gamma}_{\mathbf{1}}\right)$. According to Valiant [Val76], an EUG $U_{3}\left(\Gamma_{1}\right)$ with 3 poles contains only 3 connected poles (used as recursion base in Sect. 2.2). An optimal permutation network $P(3)$ that achieves the lower bound has 3 nodes as well. This implies that $\operatorname{size}\left(B_{3}\right)=2 \cdot P(3)+$ $\operatorname{size}\left(U_{3}\left(\Gamma_{1}\right)\right)+(3-1)=11$. Then, the size of the UC becomes $\approx 2 \cdot \frac{11}{3 \log _{2} 3} n \log _{2} n \approx$ $4.627 n \log _{2} n$, which means an asymptotically by around $2.5 \%$ smaller size than that of the 4-way UC.

However, there is a flaw in this initial design. Valiant's $U_{3}\left(\Gamma_{1}\right)$ only works as an EUG for 3 nodes under special conditions, e.g., when it is a subgraph within a larger EUG construction. There are 3 possible edges in a topologically ordered graph $G=(V, E)$ in $\Gamma_{1}(3):(1,2),(2,3)$ and $(1,3) .(1,2)$ and $(2,3)$ can be directly embedded in $U_{3}\left(\Gamma_{1}\right)$ using $\left(p_{1}, p_{2}\right)$ and $\left(p_{2}, p_{3}\right)$, respectively. $(1,3)$, however, has to be embedded as a path through node 2 , i.e., as a path $\left(\left(p_{1}, p_{2}\right),\left(p_{2}, p_{3}\right)\right)$. When $U_{3}\left(\Gamma_{1}\right)$ is a subgraph of a bigger EUG, this is possible by programming $p_{2}$ accordingly. However, when we use this $U_{3}\left(\Gamma_{1}\right)$ as a building block in our EUG construction, it cannot directly be applied. A generic $U_{3}\left(\Gamma_{1}\right)$ that can embed $(1,3)$ without going through $p_{2}$ as before has an additional Y-switching block.

We depict in Fig. 5a the 3-way body block that uses Valiant's optimized $U_{3}\left(\Gamma_{1}\right)$ in the $k$-way block design of [LMS16]. Assume that the output of pole $p_{3 i+1}$ has to be directed to pole $p_{3 i+3}$. Then, it needs to go through pole $p_{3 i+2}$, which means that the edge going in to $p_{3 i+2}$ is used by this path. However, there might be an other edge coming from the permutation network as an input to $p_{3 i+2}$, e.g., from $p_{3 i}$ from the preceding block. This cannot be directed to $p_{3 i+2}$ anymore as shown in Fig. 5a. Therefore, in the 3 -way body block construction, it does not suffice to use Valiant's optimized $U_{3}\left(\Gamma_{1}\right)$ [Val76].

Size of Body Block $\boldsymbol{B}_{\mathbf{3}}$ with Our Generic $\boldsymbol{U}_{\mathbf{3}}\left(\boldsymbol{\Gamma}_{\mathbf{1}}\right)$. In Fig. 5 b, we show the 3 -way body block with the generic $U_{3}\left(\Gamma_{1}\right)$ that allows the output from $p_{3 i+1}$ to be directed to $p_{3 i+3}$ without having to go through $p_{3 i+2}$. This results in $\operatorname{size}\left(B_{3}\right)=$ $2 \cdot P(3)+\operatorname{size}\left(U_{3}\left(\Gamma_{1}\right)\right)+(3-1)=12$, which implies that the asymptotic size of the UC is $\approx 2 \cdot \frac{12}{3 \log _{2} 3} n \log _{2} n \approx 5.047 n \log _{2} n$. Unfortunately, this is worse than the asymptotic size of the 2-way construction, and we therefore conclude that the asymptotically most efficient known UC construction is Valiant's 4-way UC construction. 


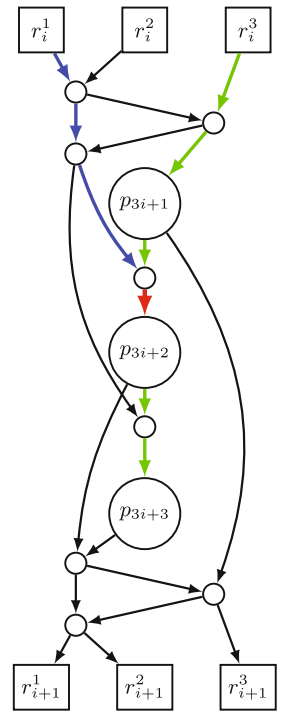

(a) Body Block with Valiant's $U_{3}\left(\Gamma_{1}\right)$

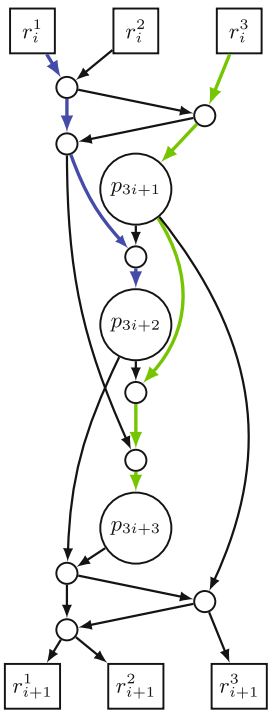

(b) Body Block with our generic $U_{3}\left(\Gamma_{1}\right)$

Fig. 5. Body block construction for our 3-way EUG $U_{n}^{(3)}\left(\Gamma_{1}\right)$.

\subsection{Hybrid Universal Circuit Construction}

In this section, we detail our hybrid UC that minimizes its size based on Valiant's 2-way and 4-way UCs, which are asymptotically the smallest UCs to date. Given the size of the input circuit $C_{u, v}^{k}$, i.e., $n=u+k+v$, we can calculate at each recursion step if it is better to create 2 subgraphs of size $\left\lceil\frac{n}{2}-1\right\rceil$ and utilize the 2way recursive skeleton, or it is more beneficial to create a 4 -way recursive skeleton with 4 subgraphs of size $\left\lceil\frac{n}{4}-1\right\rceil$.

We assume that for every $n$, we have an algorithm that computes the size ( $\left.\operatorname{size}\left(U_{n}^{\text {hybrid }}\left(\Gamma_{1}\right)\right)\right)$ of the hybrid construction for sizes smaller than $n$. We give details on how it is computed in Sect. 5. Then, Listing 2 describes the algorithm for constructing a hybrid UC, at each step based on which strategy is more efficient. We note that our hybrid construction is generic, and given multiple $k$-way UC constructions as parameter $K(K=\{2,4\}$ in our example), it minimizes the concrete size of the resulting UC.

\section{Size of UC Constructions}

Lipmaa et al.'s $k$-way UC construction is depicted in a modular manner in [LMS16, Fig. 12] and discussed briefly in Sect. 2.5 and Fig. 3. They show that a $k$-way body block consists of two permutation networks $P(k)$, an EUG for $k$ nodes, i.e., $U_{k}\left(\Gamma_{1}\right)$, and additionally, $(k-1)$ Y-switching blocks. In this section, we recapitulate the sizes (Table 2 ) of the $k$-way EUG and give an estimate for the 
Listing 2. Hybrid construction algorithm

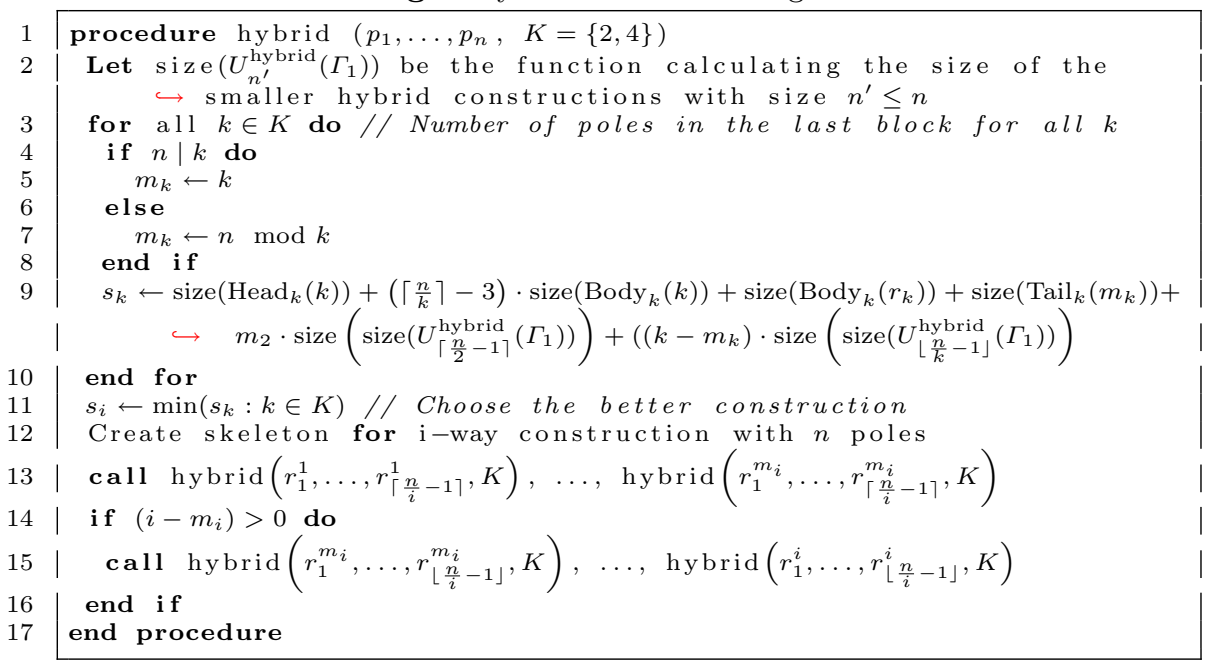

leading constant for Lipmaa et al.'s EUG construction with size $\mathcal{O}\left(n \log _{2} n\right)$, for $k \in\{2, \ldots, 8\}$. For a detailed discussion on the depth of the UCs, the reader is referred to the full version of this paper [GKS17, Sect. 5]. We conclude that the best asymptotic size is achieved by Valiant's 4-way UC. This result does not exclude the possibility for a more efficient UC in general, but it shows that the most efficient UC using Lipmaa et al.'s $k$-way UC from [LMS16] is the 4-way UC. Two $k$-way EUGs for $\Gamma_{1}(n)$ graphs build up an EUG for $\Gamma_{2}(n)$ graphs as described in Sect. 2.1. Therefore, the leading constant for the size of the UC is twice that of the EUG $U_{n}^{(k)}\left(\Gamma_{1}\right)$, which is summarized in the same table.

\subsection{Asymptotic Size of $k$-Way UC Constructions}

We review the sizes of the building blocks of a $k$-way body block, i.e., the size of an EUG $U_{k}\left(\Gamma_{1}\right)$ for $k$, and the size of a permutation network $P(k)$ with $k$ inputs and outputs, as well as the size of the resulting UCs.

Edge-Universal Graph with $k$ Poles. Valiant optimized EUGs up to size 6 by hand in [Val76]: for $k=2, U_{2}\left(\Gamma_{1}\right)$ has two poles, for $k=3$ we discussed in Sect. 4.1 that an additional node is necessary. For $k \in\{4,5,6\}$ the sizes are $\{6,10,13\}$, as shown in [KS16, Fig. 1] (note that the nodes noted as empty circles disappear in the UC ). For $k=7$ and $k=8$, we observe that Valiant's 2-way UC results in a better size than that of the 4-way UC due to the smaller permutation network and less recursion nodes. Therefore, we use these constructions to compute the size of $U_{7}\left(\Gamma_{1}\right)$ and $U_{8}\left(\Gamma_{1}\right)$. As mentioned in [LMS16], another possibility is to use the UC of $[\mathrm{KS} 08 \mathrm{~b}]$ instead of these EUGs since they have better sizes for small circuits. These UCs $U^{\mathrm{KS} 08}(k)$ are built from two smaller $U^{\mathrm{KS} 08}\left(\frac{k}{2}\right)$, a $P\left(\frac{k}{2}\right)$ and $\frac{k}{2}$ Y switches. It results in a smaller size of 21 for $k=8$. 
Table 2. The leading factors of the asymptotic $\mathcal{O}\left(n \log _{2} n\right)$ size for $k$-way edge-universal graphs $\left(U_{n}^{(k)}\left(\Gamma_{1}\right)\right)$ and universal circuits (UC) for $k \in\{2, \ldots, 8\} . n$ denotes the size of the input $\Gamma_{2}(n)$ circuit, $U_{k}\left(\Gamma_{1}\right)$ the size of Valiant's edge-universal graph with $k$ poles, $U^{\mathrm{KS} 08}(k)$ the size of the UC of $[\mathrm{KS} 08 \mathrm{~b}], P^{\mathrm{l}}(k)$ the lower bound for the size of a permutation network for $k$ nodes, and $P^{\mathrm{W}}(k)$ the size of Waksman's permutation network [Wak68]. $B_{k}^{W}$ is the size of the body block.

\begin{tabular}{r||r|r||r|r||r||r|r}
$k$ & $U_{k}\left(\Gamma_{1}\right)$ & $U^{\mathrm{KS} 08}(k)$ & $P^{\mathrm{l}}(k)$ & $P^{\mathrm{W}}(k)$ & $B_{k}^{W}$ & $U_{n}^{(k)}\left(\Gamma_{1}\right)\left(\cdot n \log _{2} n\right)$ & $\mathrm{UC}\left(\cdot n \log _{2} n\right)$ \\
\hline $\mathbf{2}$ & $\mathbf{2}$ & 2 & 1 & $\mathbf{1}$ & 5 & 2.5 & 5 \\
$\mathbf{3}$ & $\mathbf{4}$ & 6 & 3 & $\mathbf{3}$ & 12 & $\approx 2.524$ & $\approx 5.047$ \\
$\mathbf{4}$ & $\mathbf{6}$ & 7 & 5 & $\mathbf{5}$ & 19 & 2.375 & 4.75 \\
$\mathbf{5}$ & $\mathbf{1 0}$ & 11 & 7 & $\mathbf{8}$ & 30 & $\approx 2.584$ & $\approx 5.168$ \\
$\mathbf{6}$ & $\mathbf{1 3}$ & 14 & 10 & $\mathbf{1 1}$ & 40 & $\approx 2.579$ & $\approx 5.158$ \\
$\mathbf{7}$ & $\mathbf{1 9}$ & 19 & 13 & $\mathbf{1 4}$ & 53 & $\approx 2.697$ & $\approx 5.394$ \\
$\mathbf{8}$ & 23 & $\mathbf{2 1}$ & 16 & $\mathbf{1 7}$ & 62 & $\approx 2.583$ & $\approx 5.167$
\end{tabular}

Permutation Networks. Waksman in [Wak68] showed that the lower bound for the size of a permutation network is $\left\lceil\log _{2}(k !)\right\rceil$ for $k$ elements. We present this lower bound in Table 2 as $P^{\mathrm{l}}(k)$. The permutation network with the smallest size is Waksman's permutation network $P^{\mathrm{W}}(k)$ [Wak68, BD02]. For $k \in\{2,3,4\}$ its size reaches the lower bound, but for larger $k$ values, his permutation network utilizes additional nodes. Since these are the smallest existing permutation networks, we use these when calculating the size of the UC. Even with the lower bound $P^{\mathrm{l}}(k)$, for $k \in\{5,6,7,8\}$ we would have the respective leading terms $\{4.824,4.900,5.190,5\}$, which are larger than 4.75 for $k=4$.

Body Blocks. A body block $B_{k}^{W}$ is built of $(k-1)$ Y-switching blocks, an EUG for $k$ nodes, and two permutation networks [LMS16] (cf. Fig. 3). The size of $B_{k}^{W}$ is the sum of the sizes of its building blocks, i.e., $\operatorname{size}\left(B_{k}^{W}\right)=$ $\min \left(\operatorname{size}\left(U_{k}\left(\Gamma_{1}\right)\right), \operatorname{size}\left(U^{\mathrm{KS} 08}(k)\right)\right)+2 \cdot \operatorname{size}\left(P^{W}(k)\right)+k-1$.

Edge-Universal Graphs and Universal Circuits with $n$ Poles. The asymptotic size of EUG $U_{n}^{(k)}\left(\Gamma_{1}\right)$ is determined as size $\left(U_{n}^{(k)}\left(\Gamma_{1}\right)\right)=\frac{\operatorname{size}\left(B_{k}^{W}\right)}{k \log _{2} k} n \log _{2} n$ and the leading factor for a $\mathrm{UC}$ is twice this number.

\subsection{Concrete Size of UC Constructions}

The size of Lipmaa et al.'s $k$-way universal circuits depends on the size of their building blocks [LMS16]. More concretely, finding either better edge-universal graphs for small number of nodes or optimal permutation networks could improve the sizes of these UCs. Lipmaa et al. calculated the optimal $k$ value for minimizing the size of a $k$-way UC to be 3.147 [LMS16].

Table 2 shows that the smallest sizes are achieved by the 4 -way, followed by the 2-way UCs. The 3-way UC, as mentioned in Sect. 4.1, is less efficient due to 
the additional node in $U_{3}\left(\Gamma_{1}\right)$. We observe that the sizes grow with increasing $k$ values due to the permutation networks and EUGs.

Concrete Sizes of 4-Way and 2-Way UCs. Based on the parity (2-way UC) and the remainder modulo 4 (4-way UC), not only the size of the outest skeleton, but also that of the smaller subgraphs can be optimized. It was considered in [KS16] for the 2-way UC, and we now generalize the approach for $k$-way UCs. We provide a recursive formula for the concrete size of the optimized $k$-way EUG as follows. Let $m_{k}$ be defined as

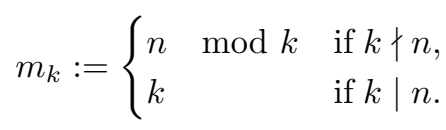

Then, given the designed Head, Body and Tail blocks with sizes shown in Table 3, we can compute the size by calculating the size of all the components of the outest skeleton, and the sizes of the smaller subgraphs with the recursive formula shown in Eq. 19. ${ }^{4}$

$$
\begin{aligned}
\operatorname{size}\left(U_{n}^{(k)}\left(\Gamma_{1}\right)\right)= & \operatorname{size}(\operatorname{Head}(k))+\left(\left\lceil\frac{n}{k}\right\rceil-3\right) \cdot \operatorname{size}(\operatorname{Body}(k)) \\
& +\operatorname{size}\left(\operatorname{Body}\left(m_{k}\right)\right)+\operatorname{size}\left(\operatorname{Tail}\left(m_{k}\right)\right) \\
& +m_{k} \cdot \operatorname{size}\left(U_{\left\lceil\frac{n}{k}-1\right\rceil}^{(k)}\left(\Gamma_{1}\right)\right)+\left(k-m_{k}\right) \cdot \operatorname{size}\left(U_{\left\lfloor\frac{n}{k}-1\right\rfloor}^{(k)}\left(\Gamma_{1}\right)\right) .
\end{aligned}
$$

Concrete Size of Our Hybrid UC. We provide a hybrid UC in Sect. 4.2 for minimizing the size of the resulting UC. This construction chooses at each step the skeleton that results in the smallest size and therefore, we provide the recursive algorithm for determining its size in Eq. 20. $\operatorname{size}\left(\operatorname{Head}_{k}(i)\right), \operatorname{size}\left(\operatorname{Tail}_{k}(i)\right)$ and

Table 3. The sizes of building blocks of the 2-way and 4-way UCs (cf. Figs. 2 and 4).

\begin{tabular}{l||cccc||cccc||cccc}
\multicolumn{1}{l||}{ Block } & \multicolumn{4}{|c||}{ Head } & \multicolumn{5}{c||}{ Body } & \multicolumn{5}{c}{ Tail } \\
$k \backslash$ Poles & 4 & 3 & 2 & 1 & 4 & 3 & 2 & 1 & 4 & 3 & 2 & 1 \\
\hline \hline Fig. & - & - & $2 \mathrm{c}$ & - & - & - & $2 \mathrm{a}$ & $2 \mathrm{~b}$ & - & - & $2 \mathrm{~d}$ & $2 \mathrm{e}$ \\
2-way & - & - & 4 & - & - & - & 5 & 5 & - & - & 4 & 1 \\
\hline Fig. & $4 \mathrm{e}$ & $4 \mathrm{~g}$ & $4 \mathrm{~h}$ & $4 \mathrm{i}$ & $4 \mathrm{a}$ & $4 \mathrm{~b}$ & $4 \mathrm{c}$ & $4 \mathrm{~d}$ & $4 \mathrm{f}$ & $4 \mathrm{~g}$ & $4 \mathrm{~h}$ & $4 \mathrm{i}$ \\
4-way & 14 & 14 & 13 & 12 & 19 & 19 & 18 & 17 & 14 & 9 & 4 & 1
\end{tabular}

\footnotetext{
${ }^{4}$ We note that for $k \geq 3$, there exist $\operatorname{Head}(k-1), \ldots, \operatorname{Head}(1)$ blocks. These are used for one $n$, e.g., $\operatorname{Head}(1)$ when $n=k+1$, and $\operatorname{Head}(k-1)$ when $n=2 k$. For simplicity, we consider these as special recursion base numbers in our calculations.
} 
$\operatorname{size}\left(\operatorname{Body}_{k}(i)\right)$ are the values from Table 3 for $k=2$ and $k=4$. The size of the hybrid $\mathrm{UC}$ is minimized as

$$
\begin{aligned}
& \operatorname{size}\left(U_{n}^{\text {hybrid }}\left(\Gamma_{1}\right)\right)=\min \left(\operatorname{size}\left(\operatorname{Head}_{k}(k)\right)+\left(\left\lceil\frac{n}{k}\right\rceil-3\right) \cdot \operatorname{size}\left(\operatorname{Body}_{k}(k)\right)\right. \\
& +\operatorname{size}\left(\operatorname{Body}_{k}\left(m_{k}\right)\right)+\operatorname{size}\left(\operatorname{Tail}_{k}\left(m_{k}\right)\right)+m_{k} \cdot \operatorname{size}\left(U_{\left\lceil\frac{n}{k}-1\right\rceil}^{\text {hybrid }}\left(\Gamma_{1}\right)\right) \\
& \left.+\left(k-m_{k}\right) \cdot \operatorname{size}\left(U_{\left\lfloor\frac{n}{k}-1\right\rfloor}^{\text {hybrid }}\left(\Gamma_{1}\right)\right) ; k \in\{2,4\}\right)
\end{aligned}
$$

which can be computed using a dynamic programming algorithm.

Improvement of 4-Way Construction. The bottom (blue) line in Fig. 6 shows the concrete improvement in percentage of the 4-way UC construction over the 2-way UC construction up to ten million nodes in the simulated input circuit. From the asymptotic leading factors in Table 2, we expect an improvement of up to $1-\frac{4.75}{5}=5 \%$. For the smallest $n$ values $(n \leq 15)$, the 2 -way $\mathrm{UC}$ is up to $33.3 \%$ better than the 4-way UC. However, from $n=212$ on, the 4-way UC construction is better, except for some short intervals as shown in Fig. 6 (the difference in these intervals, however, is at most 3.45\%). From here on, the 4 -way UC is on average $3.12 \%$ better in our experiments, where the biggest improvement is $4.48 \%$. Moreover, from $n=10885$ on, the 4-way UC always outperforms the 2 -way UC.

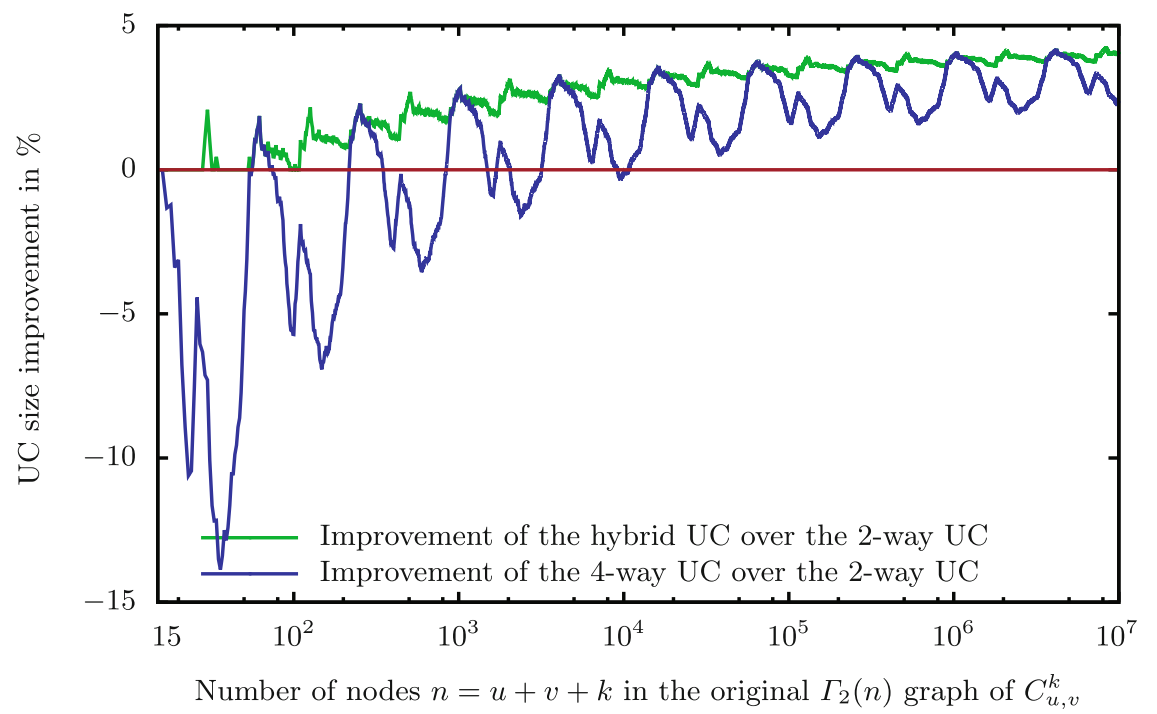

Fig. 6. Improvement of our hybrid and Valiant's 4-way UC over Valiant's 2-way UC for $15 \leq n \leq 10^{7}$ with logarithmic $x$ axis. (Color figure online) 
Improvement of Hybrid Construction. The improvement achieved by our hybrid construction (cf. Sect. 4.2) is depicted in the same Fig. 6, as the top (green) line. For some $n$ values the hybrid UC achieves the same size as the 2- or 4-way UCs, but due to its nature, it is never worse. This means that the improvement of our hybrid UC is always nonnegative, and greater than or equal to the improvement achieved by the 4 -way UC. Moreover, in most cases the hybrid UC results in better sizes than any of the other two constructions: this means that some subgraphs are created for an $n$ for which the 2-way UC is smaller, and therefore the 2 -way recursive structure is utilized. The overall improvement for all $n$ values is on average $3.65 \%$ and at most $4.48 \%$ over the 2 -way UC construction.

\section{Implementation and Evaluation}

The first implementation of Valiant's 2-way UC, along with a toolchain for PFE (cf. Sect.1.1) was given in [KS16]. The 4-way UC has smaller asymptotic size $\sim 4.75 n \log _{2} n$, but has not been implemented before due to its more complicated structure and embedding algorithm.

In this work, we improve the implementation of the open-source framework of [KS16] by using the 4-way UC construction that can directly be applied in the PFE framework. Our improved implementation is available at http://encrypto. de/code/UC. Firstly, the functionality is translated to a Boolean circuit using the Fairplay compiler [MNPS04,BNP08]. This is then transformed into a circuit in $\Gamma_{2}(n)$, i.e., with at most two incoming and outgoing wires for each gate, input and output. This is done in a preprocessing step of the framework in [KS16]. The input circuit description of our UC implementation is the same as that of the UC compiler of [KS16], and we also adapt our output UC format to that of [KS16] that includes the gate types described in Sect. 2.2. This format is compatible with the ABY framework [DSZ15] for secure function evaluation.

We discuss our implementation of Valiant's 4-way UC in Sect.6.1 and give experimental results in Sect. 6.2. For a description on how the hybrid UC can be implemented, the reader is referred to the full version [GKS17, Sect. 6.3].

\subsection{Our 4-Way Universal Circuit Implementation}

The architecture of our UC implementation is the same as that of [KS16], and therefore, we describe our UC design based on the steps described in [KS16, Fig. 6]. Our implementation gets as input a circuit with $u$ inputs, $v$ outputs and $k$ gates, and outputs a 4 -way $\mathrm{UC}$ with size $n=u+k+v$, as well as the programming $p_{f}$ corresponding to the input circuit (cf. Sect. 1).

Transforming Circuit $C_{u, v}^{k}$ into $\Gamma_{2}(u+k+v)$ Graph $G$. As a first step, we transform the circuit $C_{u, v}^{k}$ into a $\Gamma_{2}(n)$ graph $G=(V, E)$ with $n=u+k+v$ (cf. Sect. 2.1). Then, we define a topological order $\eta^{G}$ on the nodes of $G$ s.t. every input node $v_{i}$ has a topological order of $1 \leq \eta^{G}\left(v_{i}\right) \leq u$ and every output node $v_{j}$ is labelled with $u+k+1 \leq \eta^{G}\left(v_{j}\right) \leq u+k+v$. 
Table 4. Comparison of the sizes of the UCs (2-way, 4-way, and hybrid) for sample circuits from [TS15]. Bold numbers denote if the 2-way or the 4-way UC is smaller; the smallest size is always achieved by our hybrid UC. The UC generation time is given for both implemented UCs.

\begin{tabular}{|c|c|c|c|c|c|c|}
\hline \multirow[t]{2}{*}{ Circuit } & \multirow{2}{*}{$\frac{n}{u+v+k}$} & \multicolumn{3}{|c|}{ Circuit size (\#AND gates) } & \multicolumn{2}{|c|}{ UC generation (ms) } \\
\hline & & \begin{tabular}{|l} 
2-way UC \\
{$[\mathrm{KS} 16]$}
\end{tabular} & $\begin{array}{l}\text { Our 4-way } \\
\text { UC }\end{array}$ & \begin{tabular}{|l} 
Our \\
Hybrid UC
\end{tabular} & $\begin{array}{l}\text { 2-way UC } \\
{[\mathrm{KS} 16]}\end{array}$ & $\begin{array}{l}\text { Our 4-way } \\
\text { UC }\end{array}$ \\
\hline AES-non-exp & 46847 & $2.96 \cdot 10^{6}$ & $2.93 \cdot 10^{6}$ & $2.86 \cdot 10^{6}$ & 9008.9 & 10325.8 \\
\hline AES-exp & 38518 & $2.38 \cdot 10^{6}$ & $2.38 \cdot 10^{6}$ & $2.31 \cdot 10^{6}$ & 6961.7 & 8361.3 \\
\hline DES-non-exp & 31946 & $1.96 \cdot 10^{6}$ & $1.92 \cdot 10^{6}$ & $1.89 \cdot 10^{6}$ & 5563.8 & 6599.5 \\
\hline DES-exp & 32207 & $19.8 \cdot 10^{6}$ & $19.4 \cdot 10^{6}$ & $1.90 \cdot 10^{6}$ & 5654.0 & 6765.0 \\
\hline md5 & 66497 & $4.42 \cdot 10^{6}$ & $4.26 \cdot 10^{6}$ & $4.26 \cdot 10^{6}$ & 14805.5 & 14897.8 \\
\hline sha-256 & 201206 & $1.49 \cdot 10^{7}$ & $1.46 \cdot 10^{7}$ & $1.44 \cdot 10^{7}$ & 81889.1 & 57439.0 \\
\hline add_32 & 342 & $9 . \overline{5} 8 \cdot 10^{3}$ & $9.55 \cdot 10^{3}$ & $9.44 \cdot 10^{3}$ & 29.6 & 35.3 \\
\hline add_64 & 674 & $2.21 \cdot 10^{4}$ & $2.27 \cdot 10^{4}$ & $2.17 \cdot 10^{4}$ & 53.9 & 89.6 \\
\hline comp_32 & 216 & $5.53 \cdot 10^{3}$ & $5.54 \cdot 10^{3}$ & $5.49 \cdot 10^{3}$ & 17.7 & 21.2 \\
\hline mult_32×32 & 12202 & $6.54 \cdot 10^{5}$ & $6.50 \cdot 10^{5}$ & $6.35 \cdot 10^{5}$ & 1639.2 & 2177.1 \\
\hline Branching_18 & 200 & $4.92 \cdot 10^{3}$ & $5.07 \cdot 10^{3}$ & $4.88 \cdot 10^{3}$ & 21.0 & 24.2 \\
\hline CreditChecking & 82 & $1.50 \cdot 10^{3}$ & $1.51 \cdot 10^{3}$ & $1.49 \cdot 10^{3}$ & 3.1 & 12.7 \\
\hline MobileCode & 160 & $3.65 \cdot 10^{3}$ & $3.88 \cdot 10^{3}$ & $3.61 \cdot 10^{3}$ & 10.6 & 29.0 \\
\hline
\end{tabular}

Creating an EUG $U_{n}^{(4)}\left(\Gamma_{2}\right)$ for $\Gamma_{2}(n)$ Graphs. An EUG $U_{n}^{(4)}\left(\Gamma_{2}\right)$ is constructed by creating two instances of $U_{n}^{(4)}\left(\Gamma_{1}\right)$ as shown in Sect. 2.2. The two instances get merged to $U_{n}^{(4)}\left(\Gamma_{2}\right)$ so that one builds the left inputs and outputs and the other builds the right inputs and outputs of the gates (based on the two-coloring of $G$ ). We create the EUGs with Valiant's 4-way EUG [Val76] with our optimized blocks from Sect. 3.1 (cf. Fig. 4).

Programming $U_{n}^{(4)}\left(\Gamma_{2}\right)$ to Compute $C_{u, v}^{k}$. We edge-embed graph $G$ into $U_{n}^{(4)}\left(\Gamma_{2}\right)$ as described in Sect. 3.1. [KS16] use their supergraph construction to define the paths between the poles uniquely for Valiant's 2-way EUG. We modify this supergraph as described in Sect. 3.1 for Valiant's 4-way EUG and perform the edge-embedding as described in Listing 1. The programming bits of the nodes are set during the edge-embedding process along the paths between the poles. The block edge-embedding is done by analyzing the possible input values and defining the valid paths as described in Sect. 3.1.

Outputting a Universal Circuit with Its Programming. As a final step, EUG $U_{n}^{(4)}\left(\Gamma_{2}\right)$ is topologically ordered and output in the UC format of [KS16]. The programming bits $p_{f}$ defined by the embedding are also output in a separate file based on the topological order. 


\subsection{Our Experimental Results}

In order to show the improvement of our method, we ran experiments on a Desktop PC, equipped with an Intel Haswell i7-4770K CPU with $3.5 \mathrm{GHz}$ and $16 \mathrm{~GB}$ RAM, and provide our results in Table 4. To compare with the runtime of the UC compiler of [KS16], we ran the same experiments on the same platform using their 2-way UC implementation.

As [KS16], we use a set of real-life circuits from [TS15] for our benchmarks, and compare the sizes of the resulting circuits and the generation and embedding runtimes. We can see that from the 2 -way and 4 -way UC constructions, the 4-way UC, as expected, is always smaller for large circuits than the 2-way UC. However, it is sometimes better even for small circuits, e.g., for 32-bit addition with $n=342$. The hybrid construction always provides the smallest UC for our example circuits.

In the last two columns, we report the runtime of the UC compiler of [KS16] and our 4-way UC implementation for generating and programming the universal circuit corresponding to the example circuits. Table 4 shows that the differences in runtime are not significant, and due to its more complicated structure, the 4way UC takes more time to generate and program in general. However, we can see from the largest example with more than 200000 nodes that asymptotically, the 4-way UC results in a runtime improvement as well, as less nodes need to be programmed.

Acknowledgements. This work has been co-funded by the German Federal Ministry of Education and Research (BMBF) and the Hessen State Ministry for Higher Education, Research and the Arts (HMWK) within CRISP and by the DFG as part of project E3 within CROSSING. We thank the reviewers of ASIACRYPT'17 for their helpful comments.

\section{References}

[AMPR14] Afshar, A., Mohassel, P., Pinkas, B., Riva, B.: Non-interactive secure computation based on cut-and-choose. In: Nguyen, P.Q., Oswald, E. (eds.) EUROCRYPT 2014. LNCS, vol. 8441, pp. 387-404. Springer, Heidelberg (2014). https://doi.org/10.1007/978-3-642-55220-5_22

[Att14] Attrapadung, N.: Fully secure and succinct attribute based encryption for circuits from multi-linear maps. Cryptology ePrint Archive, Report 2014/772 (2014). http://ia.cr/2014/772

[BBKL17] Bicer, O., Bingol, M.A., Kiraz, M.S., Levi, A.: Towards practical PFE: an efficient 2-party private function evaluation protocol based on half gates. Cryptology ePrint Archive, Report 2017/415 (2017). http://ia.cr/2017/ 415

[BD02] Beauquier, B., Darrot, É.: On arbitrary size Waksman networks and their vulnerability. Parallel Proces. Lett. 12(3-4), 287-296 (2002) 
[BFK+09] Barni, M., Failla, P., Kolesnikov, V., Lazzeretti, R., Sadeghi, A.-R., Schneider, T.: Secure evaluation of private linear branching programs with medical applications. In: Backes, M., Ning, P. (eds.) ESORICS 2009. LNCS, vol. 5789, pp. 424-439. Springer, Heidelberg (2009). https://doi. org/10.1007/978-3-642-04444-1_26

[BNP08] Ben-David, A., Nisan, N., Pinkas, B.: FairplayMP: a system for secure multi-party computation. In: CCS 2008, pp. 257-266. ACM (2008)

[BOKP15] Banescu, S., Ochoa, M., Kunze, N., Pretschner, A.: Idea: benchmarking indistinguishability obfuscation - a candidate implementation. In: Piessens, F., Caballero, J., Bielova, N. (eds.) ESSoS 2015. LNCS, vol. 8978, pp. 149-156. Springer, Cham (2015). https://doi.org/10.1007/ 978-3-319-15618-7_12

[BPSW07] Brickell, J., Porter, D.E., Shmatikov, V., Witchel, E.: Privacy-preserving remote diagnostics. In: CCS 2007, pp. 498-507. ACM (2007)

[DSZ15] Demmler, D., Schneider, T., Zohner, M.: ABY - a framework for efficient mixed-protocol secure two-party computation. In: NDSS 2015. The Internet Society (2015). Code: http://encrypto.de/code/ABY

[FAZ05] Frikken, K.B., Atallah, M.J., Zhang, C.: Privacy-preserving credit checking. In: Electronic Commerce (EC 2005), pp. 147-154. ACM (2005)

[FGP14] Fiore, D., Gennaro, R., Pastro, V.: Efficiently verifiable computation on encrypted data. In: CCS 2015, pp. 844-855. ACM (2014)

[FVK+15] Fisch, B., Vo, B., Krell, F., Kumarasubramanian, A., Kolesnikov, V., Malkin, T., Bellovin, S.M.: Malicious-client security in blind seer: a scalable private DBMS. In: IEEE S\&P 2015, pp. 395-410. IEEE (2015)

[GGH+13a] Garg, S., Gentry, C., Halevi, S., Raykova, M., Sahai, A., Waters, B.: Candidate indistinguishability obfuscation and functional encryption for all circuits. In: FOCS 2013, pp. 40-49. IEEE (2013)

[GGH+13b] Garg, S., Gentry, C., Halevi, S., Sahai, A., Waters, B.: Attribute-based encryption for circuits from multilinear maps. In: Canetti, R., Garay, J.A. (eds.) CRYPTO 2013. LNCS, vol. 8043, pp. 479-499. Springer, Heidelberg (2013). https://doi.org/10.1007/978-3-642-40084-1_27

[GHV10] Gentry, C., Halevi, S., Vaikuntanathan, V.: $i$-hop homomorphic encryption and rerandomizable Yao circuits. In: Rabin, T. (ed.) CRYPTO 2010. LNCS, vol. 6223, pp. 155-172. Springer, Heidelberg (2010). https://doi. org/10.1007/978-3-642-14623-7_9

[GKS17] Günther, D., Kiss, Á., Schneider, T.: More efficient universal circuit constructions. Cryptology ePrint Archive, Report 2017/798 (2017). http://ia. cr $/ 2017 / 798$

[HKK+14] Huang, Y., Katz, J., Kolesnikov, V., Kumaresan, R., Malozemoff, A.J.: Amortizing garbled circuits. In: Garay, J.A., Gennaro, R. (eds.) CRYPTO 2014. LNCS, vol. 8617, pp. 458-475. Springer, Heidelberg (2014). https:// doi.org/10.1007/978-3-662-44381-1_26

[Kő31] Kőnig, D.: Gráfok és mátrixok. Matematikai és Fizikai Lapok 38, 116-119 (1931)

[KM11] Katz, J., Malka, L.: Constant-round private function evaluation with linear complexity. In: Lee, D.H., Wang, X. (eds.) ASIACRYPT 2011. LNCS, vol. 7073, pp. 556-571. Springer, Heidelberg (2011). https://doi.org/10.1007/ 978-3-642-25385-0_30

[KR11] Kamara, S., Raykova, M.: Secure outsourced computation in a multitenant cloud. In: IBM Workshop on Cryptography and Security in Clouds (2011) 
[KS08a] Kolesnikov, V., Schneider, T.: Improved garbled circuit: free XOR gates and applications. In: Aceto, L., Damgård, I., Goldberg, L.A., Halldórsson, M.M., Ingólfsdóttir, A., Walukiewicz, I. (eds.) ICALP 2008. LNCS, vol. 5126, pp. 486-498. Springer, Heidelberg (2008). https://doi.org/10.1007/ 978-3-540-70583-3_40

[KS08b] Kolesnikov, V., Schneider, T.: A practical universal circuit construction and secure evaluation of private functions. In: Tsudik, G. (ed.) FC 2008. LNCS, vol. 5143, pp. 83-97. Springer, Heidelberg (2008). https://doi.org/ 10.1007/978-3-540-85230-8_7

[KS16] Kiss, A., Schneider, T.: Valiant's universal circuit is practical. In: Fischlin, M., Coron, J.-S. (eds.) EUROCRYPT 2016. LNCS, vol. 9665, pp. 699-728. Springer, Heidelberg (2016). https://doi.org/10.1007/ 978-3-662-49890-3_27

[LMS16] Lipmaa, H., Mohassel, P., Sadeghian, S.S.: Valiant's universal circuit: improvements, implementation, and applications. Cryptology ePrint Archive, Report 2016/017 (2016). http://ia.cr/2016/017

[LP09] Lovász, L., Plummer, M.D.: Matching Theory. AMS Chelsea Publishing Series. American Mathematical Society, Providence (2009)

[LR15] Lindell, Y., Riva, B.: Blazing fast 2PC in the offline/online setting with security for malicious adversaries. In: CCS 2015, pp. 579-590. ACM (2015)

[MNPS04] Malkhi, D., Nisan, N., Pinkas, B., Sella, Y.: Fairplay - secure two-party computation system. In: USENIX Security 2004, pp. 287-302. USENIX (2004)

[MS13] Mohassel, P., Sadeghian, S.: How to hide circuits in MPC an efficient framework for private function evaluation. In: Johansson, T., Nguyen, P.Q. (eds.) EUROCRYPT 2013. LNCS, vol. 7881, pp. 557-574. Springer, Heidelberg (2013). https://doi.org/10.1007/978-3-642-38348-9_33

[MSS14] Mohassel, P., Sadeghian, S., Smart, N.P.: Actively secure private function evaluation. In: Sarkar, P., Iwata, T. (eds.) ASIACRYPT 2014. LNCS, vol. 8874, pp. 486-505. Springer, Heidelberg (2014). https://doi.org/10.1007/ 978-3-662-45608-8_26

[NPS99] Naor, M., Pinkas, B., Sumner, R.: Privacy preserving auctions and mechanism design. In: ACM Conference on Electronic Commerce (EC 1999), pp. 129-139. ACM (1999)

[NSMS14] Niksefat, S., Sadeghiyan, B., Mohassel, P., Sadeghian, S.S.: ZIDS: a privacy-preserving intrusion detection system using secure two-party computation protocols. Comput. J. 57(4), 494-509 (2014)

[OI05] Ostrovsky, R., Skeith, W.E.: Private searching on streaming data. In: Shoup, V. (ed.) CRYPTO 2005. LNCS, vol. 3621, pp. 223-240. Springer, Heidelberg (2005). https://doi.org/10.1007/11535218_14

$[$ PKV+14] Pappas, V., Krell, F., Vo, B., Kolesnikov, V., Malkin, T., Geol Choi, S., George, W., Keromytis, A.D., Bellovin, S.: Blind seer: a scalable private DBMS. In: IEEE S\&P 2014, pp. 359-374. IEEE (2014)

[Sch08] Schneider, S.: Practical secure function evaluation. Master's thesis, University Erlangen-Nürnberg, Germany, February 2008

[Sha49] Shannon, C.: The synthesis of two-terminal switching circuits. Bell Labs Tech. J. 28(1), 59-98 (1949)

[SS08] Sadeghi, A.-R., Schneider, T.: Generalized universal circuits for secure evaluation of private functions with application to data classification. In: Lee, P.J., Cheon, J.H. (eds.) ICISC 2008. LNCS, vol. 5461, pp. 336-353. Springer, Heidelberg (2009). https://doi.org/10.1007/978-3-642-00730-9_21 
[TS15] Tillich, S., Smart, N.: Circuits of basic functions suitable for MPC and FHE (2015). https://www.cs.bris.ac.uk/Research/CryptographySecurity/ $\mathrm{MPC} /$

[Val76] Valiant, L.G.: Universal circuits (preliminary report). In: STOC 1976, pp. 196-203. ACM (1976)

[Wak68] Waksman, A.: A permutation network. J. ACM 15(1), 159-163 (1968)

[Weg87] Wegener, I.: The complexity of Boolean functions. Wiley-Teubner (1987)

[Yao86] Yao, A.C.-C.: How to generate and exchange secrets (extended abstract). In: FOCS 1986, pp. 162-167. IEEE (1986)

[Zim15] Zimmerman, J.: How to obfuscate programs directly. In: Oswald, E., Fischlin, M. (eds.) EUROCRYPT 2015. LNCS, vol. 9057, pp. 439-467. Springer, Heidelberg (2015). https://doi.org/10.1007/ 978-3-662-46803-6_15 